SUPPORTING INFORMATION

\title{
Iron Acquisition Systems of Gram-negative Bacterial Pathogens Define TonB-Dependent Pathways to Novel Antibiotics
}

Phillip E. Klebba ${ }^{1 *}$, Salete M. C. Newton ${ }^{1}$, David A. Six ${ }^{2}$, Ashish Kumar ${ }^{1}$, Taihao Yang ${ }^{1}$, Brittany L. Nairn ${ }^{4}$, Colton Munger ${ }^{1}$ and Somnath Chakravorty ${ }^{3}$

${ }^{1}$ Department of Biochemistry and Molecular Biophysics, Kansas State University, Manhattan KS, 66506 USA

${ }^{2}$ Venatorx Pharmaceuticals, Inc., 30 Spring Mill Drive, Malvern, PA 19355, USA

3 Jacobs School of Medicine and Biomedical Sciences, SUNY Buffalo, Buffalo, NY, USA

${ }^{4}$ Department of Biological Sciences, Bethel University, 3900 Bethel Dr., St. Paul, MN 55112, USA

* Corresponding Author: Phillip E. Klebba, Ph. D. Department of Biochemistry \& Molecular

Biophysics, Kansas State University, Manhattan, KS 66502, United States; email: peklebba@ksu.edu 


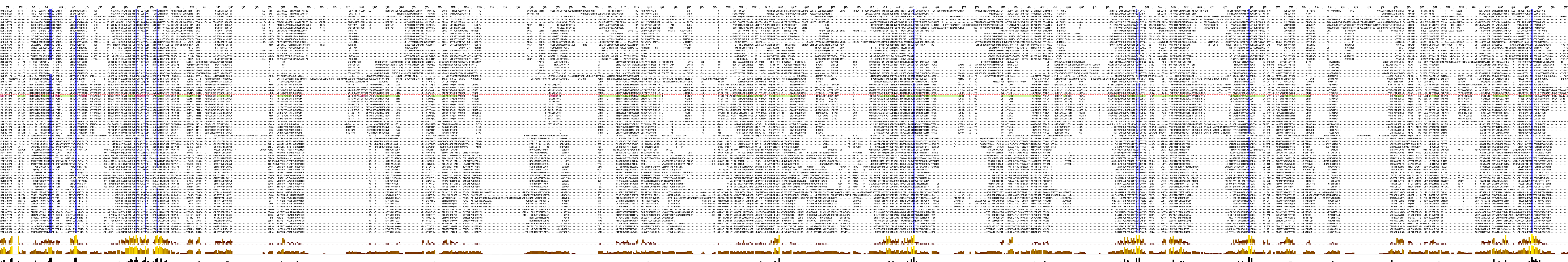




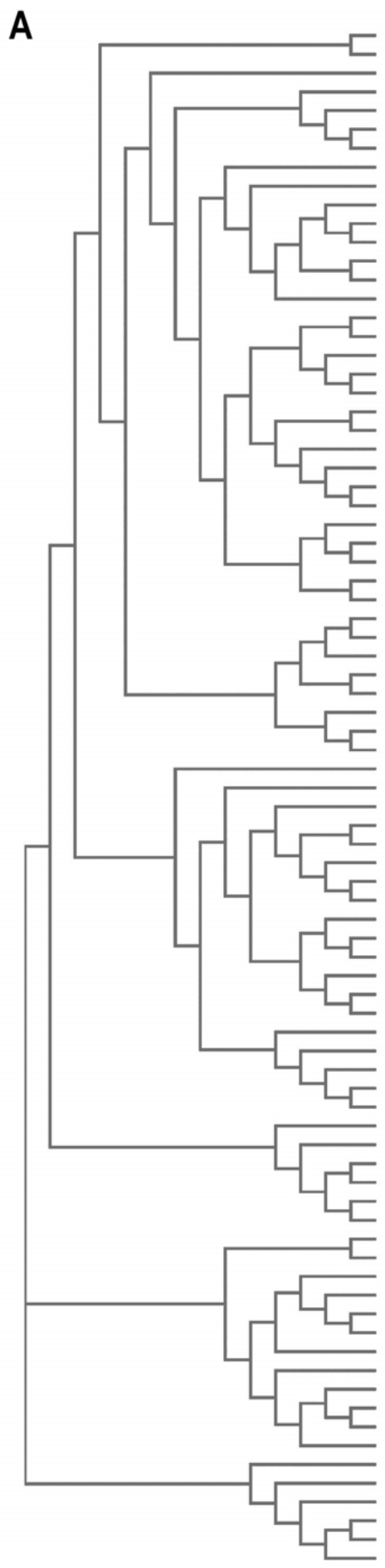

YpelGP1 0.41368

AbaBauA 0.20879

PaePupA 0.34664

PaeFptA 0.3369

PaeFhuE 0.26807

EcoFhuE 0.25152

KpnFhuE 0.25404

YpeFhuE 0.31686

BpeFauA 0.31124

PaeFpvA 0.32152

AbaLGP3 0.35834

AbaFhuA 0.34883

YpeFhuA 0.35956

AbaLGP4 0.33655

AbaLGP5 0.31947

AbaPiuA 0.28895

PaePiuD 0.28309

AbaFiu 0.31244

EcoFiu 0.11452

KpnFiu 0.0055

PaePiuA 0.00501

PaeFhuA 0.17699

EcoFhuA 0.05189

KpnFhuA 0.05252

KpnLGP2 0.29806

PaeFoxA 0.29681

EcoFyuA 0.00132

YpePsn 0.00165

KpnFyuA 0.00332

PaeFvbA 0.37235

YpeLGP2 0.36129

ECOFecA 0.40209

EcoYncD 0.11129

KpnYncD 0.10728

AbaLGP1 0.41104

AbaFepA 0.25181

PaePfeA 0.18684

KpnFep4A 0.00653

PaeFepA2 0.00544

EcoFepA 0.08878

KpnFepA1 0.00325

PaeFepA1 0.00349

PaelroN 0.02035

AbaPirA 0.00058

PaePirA 0.00076

KpnFepA2 0.17014

EcolroN 0.03684

KpniroN 0.04051

YpeLGP3 0.3224

YpeCir 0.31204

EcoCir 0.0944

KpnCirA 0.00888

PaeCirA 0.00941

EcoLGP1 0.39708

AbaBtuB 0.37505

KpnBtuB 0.0379

PaeBtuB 0.03727

EcoBtuB 0.17412

YpeBtuB 0.16953

AbaLGP2 0.27519

AbaLGP6 0.26384

KpnLGP1 0.38578

PaeHasR 0.25321

YpeHasR 0.22316

SmaHasR 0.21629

PaelGP1 0.39253

NmeFrpB 0.40618

YpeChuA 0.15078

EcoChuA 0.00213

KpnChuA 0.00276

PaeChuA 0.36892

AbaBinH 0.34717

PaeChtA 0.26153

YpelutA 0.15809

EcolutA 0.00068

KpnlutA 0.00068

PaelutA 0.1353

B
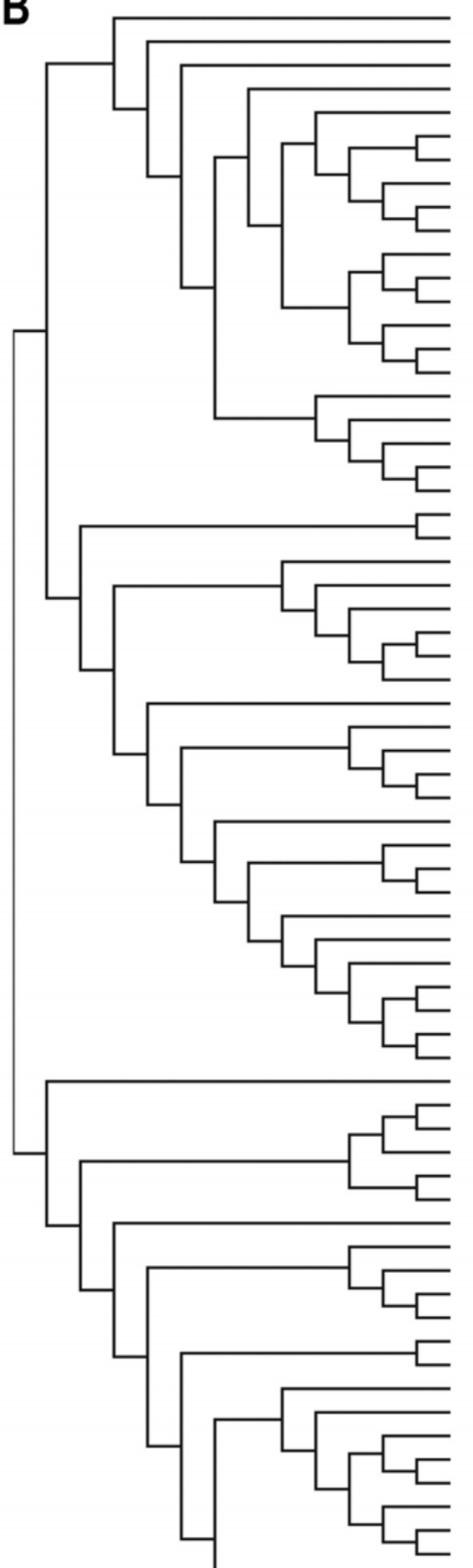

YpeLGP1 0.43889

AbaLGP1 0.436655

EcoLGP2 0.432855

AbaFepA 0.284114

PaePfeA 0.204775

KpnFep4A 0.00598404

PaeFepA2 0.00598404

EcoFepA 0.0987197

KpnFepA1 0.00336927

PaeFepA1 0.00336927

PaelroN 0.0212264

AbaPirA 0.000673854

PaePirA 0.000673854

KpnFepA2 0.182975

EcolroN 0.0413793

KpnlroN 0.0413793

YpeLGP3 0.360295

YpeCir 0.345635

EcoCir 0.104262

KpnCirA 0.00913242

PaeCirA 0.00913242

AbaLGP2 0.379203

AbaLGP6 0.379203

AbaBfnH 0.378137

PaeChtA 0.276443

YpelutA 0.162879

EcolutA 0.000682128

KpnlutA 0.000682128

PaelutA 0.138546

KpnLGP1 0.437868

PaeLGP1 0.4244

PaeHasR 0.290882

YpeHasR 0.245783

SmaHasR 0.245783

NmeFrpB 0.438649

YpeChuA 0.158869

EcoChuA 0.00244698

KpnChuA 0.00244698

EcolGP1 0.427377

PaeChuA 0.422276

AbaBtuB 0.404203

KpnBtuB 0.0400327

PaeBtuB 0.0400327

EcoBtuB 0.181596

YpeBtuB 0.181596

EcoYddB 0.442123

EcoFyuA 0.00148588

YpePsn 0.00148588

KpnFyuA 0.00297177

PaeFvbA 0.406522

YpeLGP2 0.406522

EcoFecA 0.438458

KpnFcuA 0.34817

AbaFbsN 0.330645

AbaBauA17978 0.228966

AbaBauA 0.228966

EcoYncD 0.111429

KpnYncD 0.111429

PaePupA 0.372268

PaeFptA 0.366927

PaeFhuE 0.286806

EcoFhuE 0.262689

KpnFhuE 0.262689

PaeFpvA 0.339536

YpeFhuE 0.324932

BpeFauA 0.324932

AbaLGP3 0.419379

AbaPiuA 0.307065

PaePiuD 0.307065

AbaFiu 0.355227

EcoFiu 0.115132

KpnFiu 0.00525624

PaePiuA 0.00525624

PaeFhuA 0.19898

EcoFhuA 0.0562249

KpnFhuA 0.0562249

KpnLGP2 0.313739

PaeFoxA 0.313739

YpeFhuA 0.391917

AbaFhuA 0.38278

AbaLGP4 0.350575

AbaLGP5 0.350575

Figure S2. Relationships of LGPs from CRE/ESKAPE and other Gram (-) pathogens. We analyzed NCBI FASTA protein sequences (below; red residues designate signal sequences) by CLUSTAL $\Omega$ using the BLOSUM62 matrix. A. Cladogram. The branching tree estimates the nearest-neighbor-joining patterns. B. Phylogram. The branching tree estimates phylogenetic relationships. The numerical scores estimate phylogenetic distance. 
$>$ ECOFecA

MTPLRVFRKTTPLVNTIRLSLLPLAGLSFSAFAAQVNIAPGSLDKALNQYAAHSGFTLSVDASLTRGKQS NGLHGDYDVESGLQQLLDGSGLQVKPLGNNSWTLEPAPAPKEDALTVVGDWLGDARENDVFEHAGARDVI RREDFAKTGATTMREVLNRIPGVSAPENNGTGSHDLAMNFGIRGLNPRLASRSTVLMDGIPVPFAPYGQP QLSLAPVSLGNMDAIDVVRGGGAVRYGPQSVGGVVNFVTRAIPQDFGIEAGVEGQLSPTSSQNNPKETHN LMVGGTADNGFGTALLYSGTRGSDWREHSATRIDDLMLKSKYAPDEVHTFNSLLQYYDGEADMPGGLSRA DYDADRWQSTRPYDRFWGRRKLASLGYQFQPDSQHKFNIQGFYTQTLRSGYLEQGKRITLSPRNYWVRGI EPRYSQIFMIGPSAHEVGVGYRYLNESTHEMRYYTATSSGQLPSGSSPYDRDTRSGTEAHAWYLDDKIDI GNWTITPGMRFEHIESYQNNAITGTHEEVSYNAPLPALNVLYHLTDSWNLYANTEGSFGTVQYSQIGKAV QSGNVEPEKARTWELGTRYDDGALTAEMGLFLINFNNQYDSNQTNDTVTARGKTRHTGLETQARYDLGTL TPTLDNVSIYASYAYVNAEIREKGDTYGNLVPFSPKHKGTLGVDYKPGNWTFNLNSDFQSSQFADNANTV KESADGSTGRIPGFMLWGARVAYDFGPQMADLNLAFGVKNIFDQDYFIRSYDDNNKGIYAGQPRTLYMQG SLKF

\section{$>$ EcoFepA}

MNKKIHSLALLVNLGIYGVAQAQEPTDTPVSHDDTIVVTAAEQNLQAPGVSTITADEIRKNPVARDVSKI IRTMPGVNLTGNSTSGQRGNNRQIDIRGMGPENTLILIDGKPVSSRNSVRQGWRGERDTRGDTSWVPPEM IERIEVLRGPAAARYGNGAAGGVVNI ITKKGSGEWHGSWDAYFNAPEHKEEGATKRTNFSLTGPLGDEFS FRLYGNLDKTQADAWDINQGHQSARAGTYATTLPAGREGVINKDINGVVRWDFAPLQSLELEAGYSRQGN LYAGDTQNTNSDSYTRSKYGDETNRLYRQNYALTWNGGWDNGVTTSNWVQYEHTRNSRIPEGLAGGTEGK FNEKATQDFVDIDLDDVMLHSEVNLPIDFLVNQTLTLGTEWNQQRMKDLSSNTQALTGTNTGGAIDGVST TDRSPYSKAEIFSLFAENNMELTDSTIVTPGLRFDHHSIVGNNWSPALNISQGLGDDFTLKMGIARAYKA PSLYQTNPNYILYSKGQGCYASAGGCYLQGNDDLKAETSINKEIGLEFKRDGWLAGVTWFRNDYRNKIEA GYVAVGQNAVGTDLYQWDNVPKAVVEGLEGSLNVPVSETVMWTNNITYMLKSENKTTGDRLSIIPEYTLN STLSWQAREDLSMQTTFTWYGKQQPKKYNYKGQPAVGPETKEISPYSIVGLSATWDVTKNVSLTGGVDNL FDKRLWRAGNAQTTGDLAGANYIAGAGAYTYNEPGRTWYMSVNTHF

\section{$>$ ECOFhuA}

MARSKTAQPKHSLRKIAVVVATAVSGMSVYAQAAVEPKEDTITVTAAPAPQESAWGPAATIAARQSATGT KTDTPIQKVPQSISVVTAEEMALHQPKSVKEALSYTPGVSVGTRGASNTYDHLIIRGFAAEGQSQNNYLN GLKLQGNFYNDAVIDPYMLERAEIMRGPVSVLYGKSSPGGLLNMVSKRPTTEPLKEVQFKAGTDSLFQTG FDFSDSLDDDGVYSYRLTGLARSANAQQKGSEEQRYAIAPAFTWRPDDKTNFTFLSYFQNEPETGYYGWL PKEGTVEPLPNGKRLPTDFNEGAKNNTYSRNEKMVGYSFDHEFNDTFTVRQNLRFAENKTSQNSVYGYGV CSDPANAYSKQCAALAPADKGHYLARKYVVDDEKLQNFSVDTQLQSKFATGDIDHTLLTGVDFMRMRNDI NAWFGYDDSVPLLNLYNPVNTDFDFNAKDPANSGPYRILNKQKQTGVYVQDQAQWDKVLVTLGGRYDWAD QESLNRVAGTTDKRDDKQFTWRGGVNYLFDNGVTPYFSYSESFEPSSQVGKDGNIFAPSKGKQYEVGVKY VPEDRPIVVTGAVYNLTKTNNLMADPEGSFFSVEGGEIRARGVEIEAKAALSASVNVVGSYTYTDAEYTT DTTYKGNTPAQVPKHMASLWADYTFFDGPLSGLTLGTGGRYTGSSYGDPANSFKVGSYTVVDALVRYDLA RVGMAGSNVALHVNNLFDREYVASCFNTYGCFWGAERQVVATATFRF

\section{$>$ ECOFiu}

MENNRNFPARQFHSLTFFAGLCIGITPVAQALAAEGQTNADDTLVVEASTPSLYAPQQSADPKFSRPVAD TTRTMTVISEQVIKDQGATNLTDALKNVPGVGAFFAGENGNSTTGDAIYMRGADTSNSIYIDGIRDIGSV SRDTFNTEQVEVIKGPSGTDYGRSAPTGSINMISKQPRNDSGIDASASIGSAWFRRGTLDVNQVIGDTTA VRLNVMGEKTHDAGRDKVKNERYGVAPSVAFGLGTANRLYLNYLHVTQHNTPDGGIPTIGLPGYSAPSAG TAALNHSGKVDTHNFYGTDSDYDDSTTDTATMRFEHDINDNTTIRNTTRWSRVKQDYLMTAIMGGASNIT QPTSDVNSWTWSRTANTKDVSNKILTNQTNLTSTFYTGSIGHDVSTGVEFTRETQTNYGVNPVTLPAVNI YHPDSSIHPGGLTRNGANANGQTDTFAIYAFDTLQITRDFELNGGIRLDNYHTEYDSATACGGSGRGAIT CPTGVAKGSPVTTVDTAKSGNLMNWKAGALYHLTENGNVYINYAVSQQPPGGNNFALAQSGSGNSANRTD FKPQKANTSEIGTKWQVLDKRLLLTAALFRTDIENEVEQNDDGTYSQYGKKRVEGYEISVAGNITPAWQV IGGYTQQKATIKNGKDVAQDGSSSLPYTPEHAFTLWSQYQATDDISVGAGARYIGSMHKGSDGAVGTPAF TEGYWVADAKLGYRVNRNLDFQLNVYNLFDTDYVASINKSGYRYHPGEPRTFLLTANMHF

\section{$>$ EcoFhuE}

MLSTQFNRDNQYQAITKPSLLAGCIALALLPSAAFAAPATEETVIVEGSATAPDDGENDYSVTSTSAGTK MQMTQRDIPQSVTIVSQQRMEDQQLQTLGEVMENTLGISKSQADSDRALYYSRGFQIDNYMVDGIPTYFE SRWNLGDALSDMALFERVEVVRGATGLMTGTGNPSAAINMVRKHATSREFKGDVSAEYGSWNKERYVADL QSPLTEDGKIRARIVGGYQNNDSWLDRYNSEKTFFSGIVDADLGDLTTLSAGYEYQRIDVNSPTWGGLPR WNTDGSSNSYDRARSTAPDWAYNDKEINKVFMTLKQQFADTWQATLNATHSEVEFDSKMMYVDAYVNKAD GMLVGPYSNYGPGFDYVGGTGWNSGKRKVDALDLFADGSYELFGRQHNLMFGGSYSKQNNRYFSSWANIF PDEIGSFYNFNGNFPQTDWSPQSLAQDDTTHMKSLYAATRVTLADPLHLILGARYTNWRVDTLTYSMEKN 
HTTPYAGLVFDINDNWSTYASYTSIFQPQNDRDSSGKYLAPITGNNYELGLKSDWMNSRLTTTLAIFRIE QDNVAQSTGTPIPGSNGETAYKAVDGTVSKGVEFELNGAITDNWQLTFGATRYIAEDNEGNAVNPNLPRT TVKMFTSYRLPVMPELTVGGGVNWQNRVYTDTVTPYGTFRAEQGSYALVDLFTRYQVTKNFSLQGNVNNL FDKTYDTNVEGSIVYGTPRNFSITGTYQF

\section{$>$ ECOCir}

MFRLNPFVRVGLCLSAISCAWPVLAVDDDGETMVVTASSVEQNLKDAPASISVITQEDLQRKPVQNLKDV LKEVPGVQLTNEGDNRKGVSIRGLDSSYTLILVDGKRVNSRNAVFRHNDFDLNWIPVDSIERIEVVRGPM SSLYGSDALGGVVNI ITKKIGQKWSGTVTVDTTIQEHRDRGDTYNGQFFTSGPLIDGVLGMKAYGSLAKR EKDDPQNSTTTDTGETPRIEGFSSRDGNVEFAWTPNQNHDFTAGYGFDRQDRDSDSLDKNRLERQNYSVS HNGRWDYGTSELKYYGEKVENKNPGNSSPITSESNTVDGKYTLPLTAINQFLTVGGEWRHDKLSDAVNLT GGTSSKTSASQYALFVEDEWRIFEPLALTTGVRMDDHETYGEHWSPRAYLVYNATDTVTVKGGWATAFKA PSLLQLSPDWTSNSCRGACKIVGSPDLKPETSESWELGLYYMGEEGWLEGVESSVTVFRNDVKDRISISR TSDVNAAPGYQNFVGFETGANGRRIPVFSYYNVNKARIQGVETELKIPFNDEWKLSINYTYNDGRDVSNG ENKPLSDLPFHTANGTLDWKPLALEDWSFYVSGHYTGQKRADSATAKTPGGYTIWNTGAAWQVTKDVKLR AGVLNLGDKDLSRDDYSYNEDGRRYFMAVDYRF

\section{$>$ ECOBtUB}

MIKKASLLTACSVTAFSAWAQDTSPDTLVVTANRFEQPRSTVLAPTTVVTRQDIDRWQSTSVNDVLRRLP GVDITQNGGSGQLSSIFIRGTNASHVLVLIDGVRLNLAGVSGSADLSQFPIALVQRVEYIRGPRSAVYGS DAIGGVVNI ITTRDEPGTEISAGWGSNSYQNYDVSTQQQLGDKTRVTLLGDYAHTHGYDVVAYGNTGTQA QTDNDGFLSKTLYGALEHNFTDAWSGFVRGYGYDNRTNYDAYYSPGSPLLDTRKLYSQSWDAGLRYNGEL IKSQLITSYSHSKDYNYDPHYGRYDSSATLDEMKQYTVQWANNVIVGHGSIGAGVDWQKQTTTPGTGYVE DGYDQRNTGIYLTGLQQVGDFTFEGAARSDDNSQFGRHGTWQTSAGWEFIEGYRFIASYGTSYKAPNLGQ LYGFYGNPNLDPEKSKQWEGAFEGLTAGVNWRISGYRNDVSDLIDYDDHTLKYYNEGKARIKGVEATANF DTGPLTHTVSYDYVDARNAITDTPLLRRAKQQVKYQLDWQLYDFDWGITYQYLGTRYDKDYSSYPYQTVK MGGVSLWDLAVAYPVTSHLTVRGKIANLFDKDYETVYGYQTAGREYTLSGSYTF

\section{$>$ ECOYddB}

MKRVLIPGVILCGADVAQAVDDKNMYMHVFEEMTVYAPVPVPVNGNTHYTSESIERLPTGNGNISDLLRT NPAVRMDSTQSTSLNQGDIRPEKISIHGASPYQNAYLIDGISATNNLNPANESDASSATNISGMSQGYYL DVSLLDNVTLYDSFVPVEFGRFNGGVIDAKIKRFNADDSSVELGYRTTRSDWLTSHIDENNKSAFNQGSS GSTYYSPDFKKNFYTLAFNQELADNFGVTAGLSRRQSDITRADYVSNDGIVAGRAQYKNVIDTAVSKFTW FASDRFTHDLTLKYTGSSRDYNTSTFPQSDREMGNKSYGLAWDMDTQLAWAKLRTTVGWDHISDYTRHDH DIWYTELSCTYGDITGRCTRGGLGHISQAVDNYTFKTRLDWQKFTVGNVSHQPYFGAEYIYSDAWTERHN QSESYVINAAGKKTNHTIYHKGKGSLGIDNYTLYMADRISWRNVSLMPGVRYDYDNYLSNHNISPRFMTE WDIFADQTSMITAGYNRYYGGNILDMGLRDIRNSWTESVSGNKTLTRYQDLKTPYNDELAMGLQQKIGKN VIARANYVYREAHDQISKSSRTDSATKTTITEYNNDGKTKTHSFNLSFELAEPLHISQVDINPQIVFSYI KSKGNLSLNNGYEESNTGDNRVVYNGNLVSYDSVPVADFNNPLKISLNMDFTHQPSGLVWANTLTWQEAR KARI ILGKTNAQYISEYSDYKQYVDEKLDSSLTWDTRLSWTPQFLKQQNLTISADILNVLDSKTAVDTTN TGVATYASGRTFWLDVSMKF

\section{$>$ EcoIutA}

MMISKKYTLWALNPLLLTMMAPAVAQQTDDETFVVSANRSNRTVAEMAQTTWVIENAELEQQIQGGKELK DALAQLIPGLDVSSRSRTNYGMNVRGRPLVVLVDGVRLNSSRTDSRQLDSIDPFNIDHIEVISGATSLYG GGSTGGLINIVTKKGQPETMMEFEAGTKSGFSSSKDHDERIAGAVSGGNEHISGRLSVAYQKFGGWFDGN GDATLLDNTQTGLQYSDRLDIMGTGTLNIDESRQLQLITQYYKSQGDDDYGLNLGKGFSAIRGTSTPFVS NGLNSDRIPGTERHLISLQYSDSAFLGQELVGQVYYRDESLRFYPFPTVNANKQVTAFSSSQQDTDQYGM KLTLNSKPMDGWQITWGLDADHERFTSNQMFFDLAQASASGGLNNKKIYTTGRYPSYDITNLAAFLQSGY DINNLFTLNGGVRYQYTENKIDDFIGYAQQRQIAAGKATSADAIPGGSVDYDNFLFNAGLLMHITERQQA WLNFSQGVELPDPGKYYGRGIYGAAVNGHLPLTKSVNVSDSKLEGVKVDSYELGWRFTGNNLRTQIAAYY SISDKSVVANKDLTISVVDDKRRIYGVEGAVDYLIPDTDWSTGVNFNVLKTESKVNGTWQKYDVKTASPS KATAYIGWAPDPWSLRVQSTTSFDVSDAQGYKVDGYTTVDLLGSYQLPVGTLSFSIENLFDRDYTTVWGQ RAPLYYSPGYGPASLYDYKGRGRTFGLNYSVLF

\section{$>$ ECOIrON}

MRINKILWSLTVLLVGLNSQVSVAKSSDDDNDETLVVEATAEQVLKQQPGVSVITSEDIKKTPPVNDLSD I IRKMPGVNLTGNSASGTRGNNRQIDIRGMGPENTLILIDGVPVTSRNSVRYSWRGERDTRGDTNWVPPE QVERIEVIRGPAAARYGSGAAGGVVNI ITKRPTNDWHGSLSLYTNQPESSDEGATRRANFSLSGPLAGNA LTTRLYGNLNKTDADSWDINSPVGTKNAAGHEGVRNKDINGVVSWKLNPQQILDFEAGYSRQGNIYAGDT QNSSSSAVTESLAKSGKETNRLYRQNYGITHNGIWDWGQSRFGVYYEKTNNTRMNEGLSGGGEGRILAGE KFTTNRLSSWRTSGELNIPLNVMVDQTLTVGAEWNRDKLDDPSSTSLTVNDSDISGISGSAADRSSKNHS 
QISALYIEDNIEPVPGTNIIPGLRFDYLSDSGGNFSPSLNLSQELGDYFKVKAGVARTFKAPNLYQSSEG YLLYSKGNGCPKDITSGGCYLIGNKDLDPEISVNKEIGLEFTWEDYHASVTYFRNDYQNKIVAGDNVIGQ TASGAYILKWQNGGKALVDGIEASMSFPLVKDRLNWNTNATWMITSEQKDTGNPLSVIPKYTINNSLNWT ITQAFSASVNWTLYGRQKPRTHAETRSEDTGGLSGKELGAYSLVGTNFNYDINKNLRLNVGVSNILNKQI FRSSEGANTYNEPGRAYYAGVTASF

\section{$>$ ECOYnCD}

MKIFSVRQTVLPALLVLSPVVFAADEQTMIVSAAPQVVSELDTPAAVSVVDGEEMRLATPRINLSESLTS VPGLQVQNRQNYAQDLQLSIRGFGSRSTYGIRGIRLYVDGIPATMPDGQGQTSNIDLSSVQNVEVLRGPF SALYGNASGGVMNVTTQTGQQPPTIEASSYYGSFGSWRYGLKATGATGDGTQPGDVDYTVSTTRFTTHGY RDHSGAQKNLANAKLGVRIDEASKLSLIFNSVDIKADDPGGLTKAEWKANPQQAPRAEQYDTRKTIKQTQ AGLRYERSLSAQDDMSVMMYAGERETTQYQSIPMAPQLNPSHAGGVITLQRHYQGIDSRWTHRGELCVPV TFTTGLNYENMSENRKGYNNFRLNSGMPEYGQKGELRRDERNLMWNIDPYLQTQWQLSEKLSLDAGVRYS SVWFDSNDHYVTPGNGDDSGDASYHKWLPAGSLKYAMTDAWNIYLAAGRGFETPTINELSYRADGQSGMN FGLKPSTNDTIEIVSKTRIGDGLLSLALFQTDTDDEIVVDSSSGGRTTYKNAGKTRRQGAELAWDQRFAG DFRVNASWTWLDATYRSNVCNEQDCNGNRMPGIARNMGFASIGYVPEDGWYAGTEARYMGDIMADDENTA KAPSYTLVGLFTGYKYNYHNLTVDLFGRVDNLFDKEYVGSVIVNESNVRYYEPSPGRNYGVGMNIAWRFE

$>$ ECOFYUA

MKMTRLYPLALGGLLLPAIANAQTSQQDESTLVVTASKQSSRSASANNVSSTVVSAPELSDAGVTASDKL PRVLPGLNIENSGNMLFSTISLRGVSSAQDFYNPAVTLYVDGVPQLSTNTIQALTDVQSVELLRGPQGTL YGKSAQGGIINIVTQQPDSTPRGYIEGGVSSRDSYRSKFNLSGPIQDGLLYGSVTLLRQVDDGDMINPAT GSDDLGGTRASIGNVKLRLAPDDQPWEMGFAASRECTRATQDAYVGWNDIKGRKLSISDGSPDPYMRRCT DSQTLSGKYTTDDWVFNLISAWQQQHYSRTFPSGSLI INMPQRWNQDVQELRAATLGDARTVDMVFGLYR QNTREKLNSAYDMPTMPYLSSTGYTTAETLAAYSDLTWHLTDRFDIGGGVRFSHDKSSTQYHGSMLGNPF GDQGKSNDDQVLGQLSAGYMLTDDWRVYTRVAQGYKPSGYNIVPTAGLDAKPFVAEKSINYELGTRYETA DVTLQAATFYTHTKDMQLYSGPVGMQTLSNAGKADATGVELEAKWRFAPGWSWDINGNVIRSEFTNDSEL YHGNRVPFVPRYGAGSSVNGVIDTRYGALMPRLAVNLVGPHYFDGDNQLRQGTYATLDSSLGWQATERMN ISVYVDNLFDRRYRTYGYMNGSSAVAQVNMGRTVGINTRIDFF

\section{$>$ EcoChuA}

MSRPQFTSLRLSLLALAVSATLPTFAFATETMTVTATGNARSSFEAPMMVSVIDTSAPENQTATSATDLL RHVPGITLDGTGRTNGQDVNMRGYDHRGVLVLVDGVRQGTDTGHLNGTFLDPALIKRVEIVRGPSALLYG SGALGGVISYDTVDAKDLLQEGQSSGFRVFGTGGTGDHSLGLGASAFGRTENLDGIVAWSSRDRGDLRQS NGETAPNDESINNMLAKGTWQIDSAQSLSGLVRYYNNDAREPKNPQTVGASESSNPMVDRSTIQRDAQLS YKLAPQGNDWLNADAKIYWSEVRINAQNTGSSGEYREQITKGARLENRSTLFADSFASHLLTYGGEYYRQ EQHPGGATTGFPQAKIDFSSGWLQDEITLRDLPITLLGGTRYDSYRGSSDGYKDVDADKWSSRAGMTINP TNWLMLFGSYAQAFRAPTMGEMYNDSKHFSIGRFYTNYWVPNPNLRPETNETQEYGFGLRFDDLMLSNDA LEFKASYFDTKAKDYISTTVDFAAATTMSYNVPNAKIWGWDVMTKYTTDLFSLDVAYNRTRGKDTDTGEY ISSINPDTVTSTLNIPIAHSGFSVGWVGTFADRSTHISSSYSKQPGYGVNDFYVSYQGQQALKGMTTTLV LGNAFDKEYWSPQGIPQDGRNGKIFVSYQW

\section{$>$ ECOLGP1}

MLYNIPCRIYILSTLSLCISGIVSTATATSSETKISNEETLVVTTNRSASNLWESPATIQVIDQQTLQNS TNASIADNLQDIPGVEITDNSLAGRKQIRIRGEASSRVLILIDGQEVTYQRAGDNYGVGLLIDESALERV EVVKGPYSVLYGSQAIGGIVNFITKKGGDKLASGVVKAVYNSATAGWEESIAVQGSIGGFDYRINGSYSD QGNRDTPDGRLPNTNYRNNSQGVWLGYNSGNHRFGLSLDRYRLATQTYYEDPDGSYEAFSVKIPKLEREK VGVFYDTDVDGDYLKKIHFDAYEQTIQRQFANEVKTTQPVPSPMIQALTVHNKTDTHDKQYTQAVTLQSH FSLPANNELVTGAQYKQDRVSQRSGGMTSSKSLTGFINKETRTRSYYESEQSTVSLFAQNDWQFADHWTW TMGVRQYWLSSKLTRGDGVSYTAGIISDTSLARESASDHEMVTSTSLRYSGFDNLELRAAFAQGYVFPTL SQLFMQTSAGGSVTYGNPDLKAEHSNNFELGARYNGNQWLIDSAVYYSEAKDYIASLICDGSIVCNGNTN SSRSSYYYYDNIDRAKTWGLEISAEYNGWVFSPYISGNLIRRQYETSTLKTTNTGEPAINGRIGLKHTLV MGQANI ISDVFIRAASSAKDDSNGTETNVPGWATLNFAVNTEFGNEDQYRINLALNNLTDKRYRTAHETI PAAGFNAAIGFVWNF

\section{$>$ ECOLGP2}

MRLKKHYLCTALSLAFTQQAVAAQESDSLTVWSSPVSSTTTTVLDQPTMKALDKQNVAQALSVVPGVVLQ KSGSRNEEQVKVRGFDSRQVPVYFDGVPIYVPYDGNLDLARILTNNLGAVEVSKGYSSLLQGPNQMGGAI NITTQKPTKPLEASLGYRQGWSRSQDNAYDMHASFAASSELGYLQVSGSQLKQDFLGLPHGVNNDIAGKH GKMINSSADDKRGIVKLGFTPRENDEYTLTYIKQDGEKDNPPYSGNSGQKSRYWQWPEYDKESFYYQGTT QLNDRFTLKSRLYRDTFENTLMMYNSLADLKNKKGSYSHYSDYSDGAGLQLAADVRENDLLTFAVNWKDD VHREKGAPHAAYDRYEDRTWSLASEYQWAAADNVDVVAGISYDWRDSVEAKKHEKDGSITHYDDNNQSAF 
NWQVMGKYHFVNEDTLALSYYDRKRFPTLKERYTTSKPAYNQIAIVNPQLKPERARGVDLTWNGAFTHDW GFEVSVYYNRVSDAILSHNIDADTIQNQNSGTVDYSGLDAGIKGKISNILDVGLSYALIHADAKRKDIGK ITDLPTQTMTAWMTLKPWEPLSVTLSEEARSSSYSNSDGSQKAAGFAVTHIRADYTLGHGFSVNASVNNL FDTQYAYSEGFVEEGRNFWAGVEYTF

\begin{abstract}
>KpnFep4A
MKYTSHFPLGIVIPLLACSVPLQAAENMTEQSTPDESAATAENHEETMVITAARQNLQAPGVSTITAEEI RKHPPARDVSELIRTQPGVNLTGNSTSGQRGNNRQIDIRGMGPENTLVLVDGKPVTSRNSVRYGWRGDRD SRGDTSWVPAEMIDHIDVIRGPAAARYGNGAMGGVVNIVTKPTTPEWHGSWNTYMNAPQHRKEGATKRTN FSLNGPLSDSVSFNLWGNLSKTQADAQDINAGHEAERTGSYAGSYPAGREGVVNKDIHSKLRWEFAPMQA LEFEAGYSRQGNLYAGDTQNTNTSTLVKSMYGKETNRLYRQTYGVTWTGGWDNGVTSNSYAQYEHTRNSR MDEGLAGGTEGIFSSSEFSDIDLADVLLHSEVNIPFTLGVDQNLTLGTEWNQQRMKDGVSTTQALSYGTI DGVSATGRSPYSSAEIFSLFTEDNMALTDSTMLTPALRFDHHSIVGNNWSPSLNLSQVLTDDWTLKLGIA RAYKAPNLYQLNPNYILYSNGQGCYASSSACYLMGNSDLKAETSVNKEIGLEYKHDGYQAGITWFRNDYH NKIESGYAAVGTASNGTTNIYQWENVPKALVEGLEGTLNLPVGEAVNWSNNLTWMLQSKNKTTGDRLSVI PQFTLNSTLSWQVREDLSLQSTFTWYGRQKPKRFNYKGEAVSGSELNEVSPYSIVGLSATWDVNKNLSFT SGIDNLFDIRHYRAGNAQTTGNATTGAYLYGAGAETYNESGRTFFMSVNTHF
\end{abstract}

\begin{abstract}
>KpnFepA1
MNNRIKSLALLVNLGIYGVAFPLSA AETATDDKNSAAEETMVVTAAEQNLQAPGVSTITADEIRKRPPAR DVSEIIRTMPGVNLTGNSTSGQRGNNRQIDIRGMGPENTLILIDGKPVTSRNSVRLGWRGERDTRGDTSW VPPEIIERIEVIRGPAAARYGNGAAGGVVNIITKKTGDEWHGSWNTYMNAPEHKDEGSTKRTNFSLSGPL GGDFSFRLFGNLDKTQADAWDINQGHQSERTGIYADTLPAGREGVKNKNIDGLVRWEFAPMQSLEFEAGY SRQGNLYAGDTQNTNSNDLVKENYGKETNRLYRNTYSVTWNGAWDNGVTTSNWAQYERTRNSRKGEGLAG GTEGIFNSNQFTDIDLADVMLHSEVSIPFDYLVNQNLTLGSEWNQQRMKDNASNTQALSGGEIPGYDSTG RSPYSQAEIFSLFAENNMELTDTTMLTPALRFDHHSIVGNNWSPSLNLSQGLWDDFTLKMGIARAYKAPS LYQTNPNYILYSKGQGCYASKDGCYLQGNDDLKAETSINKEIGLEFKRDGWLAGVTWFRNDYRNKIEAGY APVYQNNKGTDLYQWENVPKAVVEGLEGTLNVPVSETVNWTNNITYMLQSKNKETGDRLSI IPEYTLNST LSWQVRDDVSLQSTFTWYGKQEPKKYNYKGQPVTGSEKNEVSPYSILGLSATWDVTKYVSLTGGVDNVFD KRHWRAGNAQTTGGATGTMYGAGAETYNESGRTWYLSVNTHF
\end{abstract}

\title{
>KpnFhuA
}

MALSNTAQPINTSLRKIAVVVATAVAGMSAYTHAAETPKKEETITVTAAPAAQESAWGPAATIAARQSAT GTKTDTPIQKVPQSISVVTAEEMALHQPRSVKEALSYTPGVAVGTRGASNTYDYLIIRGFAADGQSQNNY LDGMKMQGNFYNDAVIDPYMLERAEIMRGPVSVLYGKSSPGGLLNMVSKRPTTDPLKEIQFKVGTDSLFQ TGFDFSDAIDDDGVYSYRLTGVARSNNAQQERAKEQRYAIAPSFSWRPDDKTTFTFLSYFQNEPETGYYG WLPKEGTVEPLPNGERLPTNFNEGAKNNTYSRNQKMVGYSFDHEFNDTFTVRQNLRFAENKTSQNSVYGY GVCTDPANASNKQCAALAPADKGHYLARKYVVDNEKLQNFTVDTQLQSKFSTGEVDHILLTGVDFMRMRN DINSWFGYDDSVPLLDLYNPVNSDFDFGSKDPANSGPYQILNRQKQTGLYVQDQAQWDKVLVTLGGRYDW ADQESYNRVTNTTSKRDDTQFTWRGGVNYLFDNGVTPYFSYSESFEPASQTDAQGKLFSPSKGKQYEAGV KYVPSDRPIVVTGALYQLTKTNNLMADPAGSFFSVEGGEIRARGVELEAKAALSASVNVVGSYTYTDAEY TTDTNYKGNTPAQVPKHMASVWGDYTMYDGPLSGLTLGTGVRYTGSSYGDPANSFKVGSYTVVDALVRYD LARVGMAGSNVALHVNNLFDREYVASCFNTYGCFWGAERQVVATATFRF

\begin{abstract}
$>$ KpnIutA
MMISKKYTLWALNPLLLTMMAPAVAQQTDDETFVVSANRSNRTVAEMAQTTWVIENAELEQQIQGGKELK DALAQLIPGLDVSSRSRTNYGMNVRGRPLVVLVDGVRLNSSRTDSRQLDSIDPFNIDHIEVISGATSLYG GGSTGGLINIVTKKGQPETIMEFEAGTKSGFSSSKDHDERIAGAVSGGNEHISGRLSVAYQKFGGWFDGN GDATLLDNTQTGLQYSDRLDIMGTGTLNIDESRQLQLITQYYKSQGDDDYGLNLGKGFSAIRGTSTPFVS NGLNSDRIPGTERHLISLQYSDSAFLGQELVGQVYYRDESLRFYPFPTVNANKQVTAFSSSQQDTDQYGM KLTLNSKPMDGWQITWGLDADHERFTSNQMFFDLAQASASGGLNNKKIYTTGRYPSYDITNLAAFLQSGY DINNLFTLNGGVRYQYTENKIDDFIGYAQQRQIAAGKATSADAIPGGSVDYDNFLFNAGLLMHITERQQA WLNFSQGVELPDPGKYYGRGIYGAAVNGHLPLTKSVNVSDSKLEGVKVDSYELGWRFTGNNLRTQIAAYY SISDKSVVANKDLTISVVDDKRRIYGVEGAVDYLIPDTDWSTGVNFNVLKTESKVNGTWQKYDVKTASPS KATAYIGWAPDPWSLRVQSTTSFDVSDAQGYKVDGYTTVDLLGSYQLPVGTLSFSIENLFDRDYTTVWGQ RAPLYYSPGYGPASLYDYKGRGRTFGLNYSVLF
\end{abstract}

\section{$>$ KpnFiu}

MLITKNKPAFIMEKNASLPFGSFNSLALFTGLCLGASPATAIAAEKSVKNSEETLVVEATPPSLYSPGAS ADPKFNKPLVDTTRTITVIPEQVIKDQGVTNLTDALKNVPGVGAFYAGENGSSSTGDAIYMRGVDTSNSI YVDGIRDIGSVTRDTFNTQQVEVIKGPAGTDYGRSAPSGSINMISKQPRLDSGIDGSASIGSAWSRRGTL DLNQAFSDNAAFRLNLMGEKTHDAGRDSIENERYGIAPSLAFGLDTPTRLYLNYLHVRQNNTPDGGIPTV 
GLPGYSAPSPKYAALNSAGKVDTSNFYGTDSDYDKSTTDSGTLRFEHDLTDNTTVRNTTRWSRVKQEYLL TAVMGGASNITAPDINDVNTWSWSRLVNTKDVSNRILTNQTNITSTFNTGSIGHDVSAGVEFTRENQTNY GVNAMTAPAVNLYHPVSNLSIGGLDRNGANANGQTDTFGIYAFDTLTLTERIEVNGGLRLDNYHTKYDSA TACGGSGRGAIACPPGQSTGSPVTTVDTAKSGNLVNWKAGALYRLTEQGNIYVNYAISQQPPGGSSFALA ASGSGNSANRTDFKPQKAKSSELGTKWQIFDKRLLLSAALFRTDIENEVAANDDGTWSQYGKKRVEGYEL SVTGNLTPDWTI IAGYTQQHATVTEGQNVAQDGSSALAYTPKHAFTLWTQYQATSDLSVGGGARYVGSLR RGSDGAVGTPDHTEGYWVADAKLGYRVNSNLDLQLNMYNLFDTDYVASINKSGYRYHPGEPRTFMLTANV $\mathrm{HF}$

\begin{abstract}
>KpnFepA2
MRSPQYSVPTTLALVISSLLAPNAFAEESEQDTETMIVRSTAEEALKQQPGVSIITAEDIAKQPPVNDLS DIIRKMPGVNLTGNSASGSRGNNRQIDIRGMGPENTLILIDGVPVTSRNAVRYSWRGERDTRGDSNWVPA EMVERIEVLRGPAAARYGSGAAGGVVNIITKRPTNTWHGSLSFFTNQPENNKEGTTNRANFNLSGPLAGE ALTMRLYGNINKTEPDAWDINHAQNGSYAAGREGVRNKDINALLSWKMTPQQILDFSYAYSRQGNIYAGD TQYSNGNLSPNGLVDSLYGHETNRLYRQSWGLTYNGLWDWGQSKAGVYYEKTNNTRLQEGSTGRVEGMIN SEDYATSRLESWRTTSEFNVPFFWLADQTLTLGMEWNHDQLDDPASMQATNSNGETIPGTSGDPTQRSTK NSATLTGIYLEDNIEAVPGTNLIPGIRFDYHNQFGSNWSPSLNLSQELGDMFTLKAGIARVFKAPNLYQS SKGYLLSTRGNGCPNTIAEGSCYLLGNPDLDPEISINKEIGIEFNLNGYAAGVTWFRNDYKNKIVSGTEV LGYTSSGNNILQWQNGGKAVVEGLEGNLLIPVLRDVLSWRTNATWMLKSESKETGNPLSVIPKYTVNTML DWQVNDALSANVNWTLYGRQKPRQYAEIRNETGTLATTEVGAYSIVGIGTQYQLNRDIRLNAGISNLFDK QLYRENAGASTYNEPGRAYYAGVTLSF
\end{abstract}

\title{
>KpnFhuE
}

MHFKEIAGIYSIAVPGASGITRTFCYSLLTLCIANAVSIQSAVAAESSAAASDTFKEESLVVTADTGGDS TSNSAQSYTVPATRAGTKLNLTQRDIPQSVSVLTQQRIRDQNLQNVGDALTNVTGISASQNDSERVDFYS RGFYINNYTYDDIPSTQTGAWNFGETDGDSAIYERIDVVRGSTGLMTGTGNPGAAINMIRKKADSKMFAG NLSASYGSWDNQRYVADLSGPLNDSGTLRGRVITGYQDQDSWVDRYHKRKKFLYATVAADLTDNTTLDLG YDYQSRNTDGPTWGGLPTWYSNGSKIQYDQGDNPSADWTHYNILSRKVFANLTTNFDNGWQARVNGTHTE SDFDSKLLYVTGYPDQSTGILNSSGYGYAGWYKGLRTQTAVDAYATGPFEMFGRQHQLVAGVDYSRQRNR YLYSTSILSPAEIGNWNNWNGDVTEPDWPAWALSSEDTIRQKAGYAAARFSL IDPLSL ILGARYTQYSAH GTSADMEKNNITPYGGLVYDINENYSAFASYTSIFQPQTYRDQQGNYLKPVTGKDYEAGIKGDWDDNRIT ASLSVFRIEQDNLGQIQSDIYVNNSSENAYKASKGVVSRGVDFEINGAVTDNLKLTFGATRYVAKDADGA RTNPYMPQTSLKLFTSYNLPMLPELTIGGGVNWQNRTYKEATNPSGVTERVYQGSYPLANLFARYQVTRE LSVQGNIKNLFDREYNTNPSGGVAFNEPRNYSVSVNYSF

\begin{abstract}
$>$ KpnIroN
MGMRINKILWPSIALLAGLNSQVSVAKSYDDNDETLVVVATAEQVLKQQPGVSIITSEDIKKTPPVNDLS DIIRKMPGVNLTGNSASGTRGNNRQIDIRGMGPENTLILIDGVPVTSRNSVRYSWRGERDTRGDTNWVPP EQVERIELIRGPAAARYGSGAAGGVVNIITKRPSNDWHGSLSLYTNQPESSDEGATRRTNFSLSGPLAGD ALTMHLYGNLNKTDADSWDINSSAGTKNAAGHEGVRNKDINGVVSWKLNPQQILDFEAGYSRQGNIYAGD TQNSSSSAVTENLAKSRKETNRLYRQNYGIAHNGIWDWGQSRFGVYYEKTNNTRMNEGLSGGSEGRILAD EKFTTNRLSSWRTSGEINIPLNEPVDQTLTVGVEWRRDKLDAPSSTSLTVNDSDIGGISGSAADRSSKNH SQISALYIDDNIEPVPGTNI IPGLRFDYLNESGGNFSPSLNLSQELGDYFKVKVGIARTFKAPNLYQSSK GYLLYSKGNGCPKDITSGGCYLKGNKDLDPEISINKEIGLEFAWEDYYASVTYFRNDYQNKIVAGDNAIG QTASGAYILQWQNGGKALVDGIEASMAFPLVKGRLNWNTNATWMITSEQKDTGNPLSI IPKYTINNSLDW TITQAFSASVNWTLYGRQKPRTHAETRSEDTRGMSGKVLGAYSLVGTNFNYDINKNLRLNIGISNILDKQ IYRSSEGASTYNEPGRAYYVGAVVSF
\end{abstract}

\section{$>$ KpnFCuA}

MHTTHYSSFPLRKTLLALAIGAASQTVMAADAAAAKQPGEETLIVEANETSDFKSGGDLVVPAFLDGQIA HGGRLGMLGEQKAMDVPFNVIGYTSKLIQDQQAKTIADVVSNDAGVQAVQGYGNFAETYRIRGFKLDGDD MTMGGLAGVVPRQVMDTQMLERVEIFKGANSLLNGAASSGVGGMINLEPKRAEDLPTARVGVDYTSDSQV GGTLDLGRRFGDNNQFGARVNLVHREGEGAIDNDKRRTTLASLGLDYRGDRFRSSLDFGYQKKTFHGGTM GVNISGVDFVPALPDNSKNYSQKWGYSDIESEFGMAKAEYDLTDSWTVYSALGGQHSHEIGTYSAPKLLN KNGDATVGRLDTNRIIDAISGMGGVRGDFNTGAISHKVNLGYAAQVHTDATAWRMSARNPTTNIYDNHDV AMPDNAYFGGNYHDPLVTSRSRTQGWLLSDTLGFFNDKVLFTAAARHQKVVVRNYSNATGLEDTSSRYTQ SRWMPTFGLVYKPWEQLSLYANHTEALQPGSVAPTTAANAGQSTGIAHSKQDEVGVKIDYGTIGGSLALF EIKKPNAISDTAGNYGLDGEQRNRGVEMNVFGEPMLGLRLNASTVWLDAKQTKTAEGATDGKDAIGVANF YAVLGAEYDIKPVEGLTATARVNHSGSQYADAANTKKLDSYTTLDLGLRYRMRLNADQNEMIWRVGVTNV TNEKYWSGIDDTGTYLFEGDPRTVRVSMSYDF

\section{>KpnYncD}


MKILSVRHVALPALLLPLIAAAQAADEQTMVVTAAPTTVSELDTPAAVSVVNGDEMRQAAPRVNLSESLG AVPGLQVQNRQNYAQDLQLSIRGFGSRSTYGVRGLRIYVDGIPATMPDGQGQTSNIDIGSVDTIEVLRGP FSALYGNSSGGVINVTSQTGTQPPTVEASSYYSSFGTWHYGMKATGAVGDGSHAGDVDYTVSTNRFTTHG YRDHSGARKNLANARLGVRINDVSKLTLLLNSVDIKANDAGGLTADEWRDNPRQSPRGDQYNTRKNTRQT QAGLRYERQLSAQDDLSVMMYAGERETTQFQSIPRAPQLKPSHAGGVIDLTRHYQGIDTRLTHRGELLVP VTLTAGLDYENMSERRKGYENFVMVNGAPQYGEQGALRRNERNLMWNVDPYLQTQWQLTDKLSLDAGVRY SSVWFDSNDYYITPGNGDDSGDASYHKWLPAGSLKYALTDAWNVYLSAGRGFETPTINELSYRSDNQSGL NFGLKPSTNDTVEIGSKTRIGNGLFTAALFQTNTDNEIVVDSSSGGRTSYKNAGKTRRQGMELGLDQQFG ESWRLKAAWTWLDATYRTNVCDDASCNGNRIPGIARNMGYASFGYQPEQGWYAGSDIRYMSDIMANDENT AKAPSWTVVGLTTGYKWSYGRMDMDLFGRIDNLFDREYVGSVIVNESNGRYYEPAPGRNYGIGLNLAWRF E

\title{
>KPnFyuA
}

MKMTRLYPLALGGLLLPAIANAQTSQQDESTLVVTASKQSSRSASANNVSSTVVSAPELSDAGVTASDKL PRVLPGLNIENSGNMLFSTISLRGVSSAQNFYNPAVTLYVDGVPQLSTNTIQALTDVQSVELLRGPQGTL YGKSAQGGI INIVTQQPDSTPRGYIEGGVSSRDSYRSKFNLSGPIQDGLLYGSVTLLRQVDDGDMINPAT GSDDLGGTRASIGNVKLRLAPDDQPWEMGFAASRECTRATQDAYVGWNDIKGRKLSISDGSPDPYMRRCT DSQTLSGKYTTDDWVFNLISAWQQQHYSRTFPSGSLIVNMPQRWNQDVQELRAATLGDARTVDMVFGLYR QNTREKLNSAYDMPTMPYLSSTGYTTAETLAAYSDLTWHLTDRFDIGGGVRFSHDKSSTQYHGSMLGNPF GDQGKSNDDQVLGQLSAGYMLTDDWRVYTRVAQGYQPSGYNIVPTAGLDAKPFVAEKSINYELGTRYETA DVTLQAATFYTHTRDMQLYSGPVGMQTLSNAGKADATGVELEAKWRFAPGWSWDINGNVIRSEFTNDSEL YHGNRVPFVPRYGAGSSVNGVIDTRYGALMPRLAVNLVGPHYFDGDNQLRQGTYATLDSSLGWQATERMN ISVYVDNLFDRRYRTYGYMNGSSAVAQVNMGRTVGINTRIDFF

\begin{abstract}
>KpnCirA
MFRLNPFIRAGLSASVVSLAFPALADVNEETLVVTASATEQNVKDAPASISVITQQDLQRKPVQNLKDVL RDVPGVQLTNEGDNRKGVSIRGLSSSYTLILVDGKRVNSRNAVFRHNDFDLNWIPVDAIERIEVVRGPMS SLYGSDALGGVVNIITKKIGQKWTGTLSADTTIQEHRDRGDTYNGQFFTSGPLIDGVLGMKAYGSLAKRA KDDPQSSSNATGETPRIEGFTSRDGNVEFAWTPNENHDFTAGYGFDRQDRDSDSLDRNRLERENYSLSHN GRWDIGNSELKFYGEKVDNKNPGQSGTITSESNAIDGKYVLPLGMINQLVTFGGEWRHDKLKDPVNLSSG GQSTSASQYALFIEDEWRIIEPLALTTGIRMDDHQTYGDHWSPRAYLVYNATDTVTVKGGWATAFKAPSL LQLNPDWTTNSCRGSCSIVGNPDLKPETSESFELGLYYRGEEGWLENVEGSITTFQNNVDDMIDVLRTSS ASEAPGYPNFVGWKTVNGKRVPIFRYFNVNKARIKGVETEVKIPFGDEWKLTVNYTYNDGRDLSNGGDKP LQTLPFHTANGTLDWKPLDDWSFYVTANYTGQQRAVSATGKTPGGYTLFDVGAAWQVTKNVKLRSGVQNV GDKDLSRDDYSYTEEGRRYFMAVDYRF
\end{abstract}

\begin{abstract}
$>$ KpnChuA
MMVSVIDTSAPENQTATSATDLLRHVPGITLDGTGRTNGQDVNMRGYDHRGVLVLVDGVRQGTDTGHLNG TFLDPALIKRVEIVRGPSALLYGSGALGGVISYDTVDAKDLLQEGQSSGFRVFGTGGTGDHSLGLGASAF GRTENLDGIVAWSSRDRGDLRQSNGETAPNDESINNMLAKGTWQIDSAQSLSGLVRYYNNDAREPKNPQT VEASDSSNPMVDRSTIQRDAQLSYKLAPQGNDWLNADAKIYWSEVRINAQNTGSSGEYREQITKGTRLEN RSTLFADSFASHLLTYGGEYYRQEQHPGGATTGFPQAKIDFSSGWLQDEITLRDLPITLLGGTRYDSYRG SSDGYKDVDADKWSSRAGMTINPTNWLMLFGSYAQAFRAPTMGEMYNDSKHFSIGRFYTNYWVPNPNLRP ETNETQEYGFGLRFDDLMLSNDALEFKASYFDTKAKDYISTTVDFAAATTMSYNVPNAKIWGWDVMTKYT TDLFSLDVAYNRTRGKDTDTGEYISSINPDTVTSTLNIPIAHSGFSVGWVGTFADRSTHISSSYSKQPGY GVNDFYVSYQGQQALKGMTTTLVLGNAFDKEYWSPQGIPQDGRNGKIFVSYQW
\end{abstract}

\section{>KpnBtUB}

MIKKASLMTALSVTAFSGWAQDSNSDTLVVTANRFQQPVNTVLAPTDIVTRDDIDRWQSKDLNDVMRRLP GVDIARNGGMGQSASLYVRGTEARHVLVLIDGVPMARLGISNGVDISQIPISLVQRVEYIRGPRSAVYGS GAIGGVVNIITMTDSDRSQVNVGMGSNGYQTYDGAINKRFGDTVVTAAGAYQTTKGFNVQPNSPYSGDSD RDGYRNKLFLGGVQHKFDDNFSGFFRGYGYSANADYDQGNWGYAGGNDEDQSYTQSWDTGLHYHSGIYST QLIANYQRIKDYNYSSDAGRYAAGTTLDDMEQRYIQWGNNVVVGHGAVSGGVDWKQEKLKSSGTTSTDVY KRDTTGLYLTGQQQIDSVTLEASGREDHDEQFGWHGTWQTAAGWEFIDGYRTTLSYGTGFLAPSLGQQYG AERFGIASNPNLKPEESKQWEAGLEGLTGPVDWRLSAYRYEIQNLIDYDNNAYYNVKSATIKGLEWTGNI TTGPVEHHLTLQYVDPRDDETNKILYRRAKQQVKYELNGQVYDLGWDVTYHYIGKRYDYDYDNSRTVNMG GLSLWDVGLSYPVTSHLTVRGKIANLFDKDYETVYGYQSAGREYTLSGSYTF

\section{$>$ KpnLGP1}

MNVIKLAIGSGILLLSCGAYSQSISEKTNSDKKGAAEFSPLSVSVGKTTSEQEALEKTGATSSRTTDKNL QSLDATVRSMPGTYTQIDPGQGAISVNIRGMSGFGRVNTMVDGITQSFYGTSTSGTTTHGSTNNMAGVLI DPNLLVAVDVTRGDSSGSEGINALAGSANMRTIGVDDVIFNGNTYGLRSRFSVGSNGLGRSGMIALGGKS 
DAFTDTGSIGVMAAVSGSSVYSNFSNGSGINSKEFGYDKYMKQNPKSQLYKMDIRPDEFNSFELSARTYE NKFTRRDITSDDYYIKYHYTPFSELIDFNVTASTSRGNQKYRDGSLYTFYKTSAQNRSDALDINNTSRFT VADNDLEFMLGSKLMRTRYDRTIHSAAGDPKANQESIENNPFAPSGQQDISALYTGLKVTRGIWEADFNL NYTRNRITGYKPACDSRVICVPQGSYDIDDKEGGFNPSVQLSAQVTPWLQPFIGYSKSMRAPNIQEMFFS NSGGASMNPFLKPERAETWQAGFNIDTRDLLVEQDALRFKALAYRSRIQNYIYSESYLVCSGGRKCSLPE VIGNGWEGISDEYSDNMYIYVNSASDVIAKGFELEMDYDAGFAFGRLSFSQQQTDQPTSIASTHFGAGDI TELPRKYMTLDTGVRFFDNALTLGTIIKYTGKARRLSPDFEQDEHTGAIIKQDLPQIPTIIDLYGTYEYN RNLTLKLSVQNLMNRDYSEALNKLNMMPGLGDETHPANSARGRTWIFGGDIRF

\begin{abstract}
>KpnLGP2
MNVAISRKRPGLLYALAVALPFTAQAEETVVVTATPPASASAPTEGYSTSTSLGATKTDQPLITTAQSVS VVTRQQMADQGANTISQALEYTPGVYSSFGGGATRFDAISLRGYHGGDVDNLFLDGMRLMSDGGSHNVLQ IDPWFIERVDVIRGPSSALYGQSVPGGVVNLTSKRPQFSQQGHIRLTGGTQNTKGAAFDYTDAINDQWAW RLIGMTRSSDTQYDHTREERYAISPSLLWQPDSDTSLLLRAYLQKDPSGGYHGSLPLDGTRYAHNGRKLS PSTNEGDPGDGYQRRQQIYSYEFDHQFTDVWSVYSAGSYTHTNVSLDQVYQVGWIDDSDMLARGYSGSRG SLDGWSTDNRLRADFNTGDLAHTLILGAEYHRFRNDLWTGAGGAAPLNPFSGYTAQTGHTVTYSDDNNRR YYQTGLYLQDEMVWNRWHVDVSARYDRIVSRQVSDTQGTSNRRSDDHISGRASLLYALDNGLSPYLSYSQ AITPAMLPGADGKPLKPTTAEQVEAGLKFQPPGSSDLYSIAVYDLTQKDVATRDPNIATATYIPAGKVHS QGVELEAHHQITPQLSTIASYTWNRLRFQDTKDGTDNNTPQLTPDQMASFWARYQFPAGISVGAGVRYIG KQWADDANTARLPSVTLMDAMMRADLGVWSPTLKGAYVQVNANNIGDREYISGCYGTGNCYWGAERSVIA TVGYDF
\end{abstract}

\begin{abstract}
$>$ AbaFiu
MAKIKSRKHIATTPLVALAASLPLASHAATKEEKLTQLPTIEVTAOEIYKANTVSSPKYTQPLVDTPQTI QVIKKEILQEQGAASLVEALRNTPGITLQMGENGNTSAGDTFQMRGFSTQTSTYVDGIRDLGAVSRDVFN LEQIEVVKGPSGAEAGRGSASGYINLVTKLPKAENSREVSARYNTAENARLTADINQAISESTAIRLNVM GQDGGVEGRDVIENNGWAIAPSIAFGLDSDTRLYLYSQHIRQNNIPDGGIPTIGMKGFYGYTSSYANDKN LALRDQIREAINKSADMQKVNKALQNAPEVDRSNFYGSVNDYEDISSDMISAKIESDFSGNIKFTNISRL GKTSMKRALTGINAINAPSATVTVNPDNSYSYKFNYGSFDPNNRDTWAISRSHQGVDQENKILANTSNLN INFKTGAVEHDLVAGLEFLREQQISRTLSTQKPGTKAPTTPAANLYNPNHDDVMPELVYTGAYSDGETKT AAAYLFDTVKLLDNRLQFNGGVRLDYYHTDYEALAVATNATTLAITKTPTHLKAHDTLVSWKLGSLFKPT KNSSLYVSYAKSLTPPGSANFSLSDTGANSSAAKPQKTNHYEMGGKWDVLKNKLALTSAIYHTENENQFT QDPITKESIQEGKTRVQGVELGVVGQITDAWNISAGVAHMKTKQKNQQSFNTTTGEKTVTDSVRWSPDWT ASLWTTYDIAGFKLGLGARYVDEQKRVITDSTAPANMPKVPSYVVFDAMAGYSLNKNASVNLNVYNLADK DYISALNNGGSRFVLGQPRSAALTFNYKF
\end{abstract}

\footnotetext{
$>$ AbaBfnH

MEKMNVNNKIGVKSRNHIRSKQVLFKMTPCCLAISAIFAQQAYAETVTQTAEVSENATQKPVAQLQKIVV TATRTPKNIAEIAGTVQSIDQKQIIQQATAGRKVADILAQLVPSLASSSGTTSNYGQTMRGRNVLVMIDG VSQTGSRDVSRQLNSISPGMIERIEVISGATSIYGSGATGGIINIITKRADTSKPLSFETKVGITSSDTF RSDGLAYEVGQSVSFNKGNIDGFLGANFTSRGSQFDGNGDRISLSPWQGSTMDTDTIDVNGRLNFNLNDT QTLSFGAQYYKDKQDTDYGPDYSYLPTTSKSNDATTPTYKAIKGLKLSNPLFTERYAVNSQYQNQDFLGQ ILNVEAYYRNEKSRFFPYGLSNKSVTSVNQSQSEIEVAGLRSTMQTDLNIANRDMKITYGLDYDWEKDKQ FVDILATQYPYLVYTPTGQRKGYGPNTEIQNIGAFVQSDYAVTDKLNLQAGIRYQYIQADTDAYIPSRET TMVPAGSTHDDKPLFNLGAVYKLTDAQQVYANFSQGFSFPDVQRMLRDVSTYTVSTANLQPITVNSYELG WRLNQDDGLNLGLTGFYNTSDKTVQFNNRAAKVVDTDQRVYGAEATISYPFMENYKVGGTLGYTRGQYKD VANKWHELNSFTVAPVKGTLFAEWDNNEGYGVRVQMQAIKGTNKAYKDDRELAAFATTQDEAFQNAVKND ANSAAQIKGYTTMDVLAHFPAWKGRVDFGVYNVWNRQYRTVFAQQAAVSNANPLLAIPAEGRTYGLSYTF NY

\begin{abstract}
$>$ AbaFepA
MSKRIIQSVLSVSVLASMMSMAFAAQNEQEQAEQTLEKPAEPVKLETIFVTAEEQVKQSLGVSVITKEDL EKLPVRNDISDYVRRMPGVNLTGNSATGQRGNNRQIDIRGMGPENTLILVDGKPINSRNSVRYGWKGERD TRGDSNWVPAEAIESIEVLRGPAAARYGSGAAGGVVNIITKKVTNETHGSVEFYTSQPEDSKEGSSNRVG FNVSGPLIKDVLSYRLYGNYNKTEADDVDINKSIGSTAAGREGVKNKDISGRLAWQATDQQTVLLDISSS KQGNIYSGDSQLNANAEADILSQLIGKETNTMYRDSYALTHEGDWSWGKSKLVAQYDKTHNKRLPEGLAG SVEGKINNLDDKATSRLETLRFNGEANIPFEYYLPQVLTVGTEWVEDRFKDNVSTTQGKDSSGSGYGDQL AKGDRSKMESRIASAYIEDNLKVTDSTDVVLGLRFDDHSKSGSNWSPSLNITQKLNDYFTLKGGVAKAYK APNMYQNAEGYLLSTNGNGCPANIESRCLLQGNGDLKPETSVNKELGIQFQKDIVNASLTWFRNDYKDKI VAGTHVVGTVDGSSTNANTGAVTNTKWNILRWENTPKALIQGFEGSLGLDFGDIRWTNNFTYMMDSKDKQ TGNPLSLVPIYTINSIFDYDITDQLDVNFVFTQYGRQKSRQFAENRLESGIGSGGANSALKPSTVKSYST AGINVGYKFSDQISTRVGVSNLFDKQILRDSNSISQTYNEPGRAYYASLKYSF
\end{abstract}




\title{
$>$ AbaPiuA
}

MAQEAVSQLPTIHTKATQEESLKVDQSANSKFVAPLKDTPKSVSILSQKLIKDTNSNTLLEALRYEPGIT LGAGEGGTPFTDMPYIRGYSGQSSIYVDGVRNTTSQNRDMFAIEQVEVIKGSSSALGGGGSVGGSINLIP KVAHEGDVYQGSVQSGTDNYRHIQLDANKDFGNGIAGRVVIMGHENEKAGQSNGAEYKRVGIAPSITFGL DTATRGTLSYYYLRSNDEPDAGIPFNNANPAKPPVGVTVTPGDGKPVDVKAGTYYGWKARDFDKRENHIG TFKLEHDFNENLTLSNIATYNKSKSDYVYTNADDSKGNIYRGTVARRALSRILDTDAYSDQLSLRGKFNT GSLKHSFNVGTEWSFQETDQGVHTFTNAAGETTSTILDSNIQNCTSAAAVANGWCTSLNNPGNGAFTDKR GSITAQSTTRSHNVGIYALDSIEFNPQWLLNLGVRWDKFETEKKYNKDVDGRTPHKAGDKLESDTDYFSY QAGLVFKPTEDGSIYLSYATSANPVGVLAEGDTGSDSISDSGTASASANALKPEEARTFELGTKWDLFNN RANLTAAVFRTEKQNTRIQIDPTTTANAGKSKVDGFEISLNGKITDKWDVSTGYSYLDSEITEAAYNAVA QEGKPLPFVAKNSATLWSTYRVMPQLTLGAGVEYRDQVFVNTTAPKYLPTYTIYNAMAKYDVNKNVNLQL NINNISDKRYFTSAHAAHYAFEGNGRNAVLAINFKY

\begin{abstract}
$>$ AbaPirA
MYPQFRRGHLAAAVLFASSSLLGGQALAEDERLEELDERAESVVQLGDEVVLGTAEQELKQAPGVSIITA EDIRKRPPVNDLSEIIRTMPGVNLTGNSSSGQRGNNRQIDIRGMGPENTLILVDGKPVSSRNSVRYGWRG ERDTRGDSNWVPPEEVERIEVLRGPAAARYGSGAAGGVVNI ITKRPTDRLRGSMTVFTNIPESSKDGATR RANFSLSGPLTEALSFRAYGSANKTDSDDTDINLGHTVNPSRTVAGREGVRNRDLSGMLSWQVTPDQVVD FEAGFSRQGNIYAGDTQNNNGTANTQGLADDGAETNRMYRENYAITHNGTWSFGTSRFVAQYDSTRNNRL EEGLAGSVEGQIGADRSFSTSKLENYRLSGELNLPLHALFEQVLTVGAEWNKETLNDPSSLKQGFVGSDS LPGTPAAGSRSPKSKAEIRALYVEDNIELRPGTMLTPGLRLDDHSDFGLNWSPSLNASQTLGEYFTVKAG IARAFKAPNLYQSNPNYLLYTRGNGCPIQTSSGGCYLVGNENLDAETSVNKELGIEFRRDGWVAGLTYFR NDYKNKIVAPLDVMGQTGTGNNILQWSNAKKAVVEGLEGNLLVPLHEDLSWSTNLTYMLQSKDKDTGNPL SVIPEYTLNSTLDWQASERLSTQLTSTIYGRQEPPKHGTSRNTPVVSRKEVGTYGIWGVSAGYTFSENLS VRGGVSNLFDKRLYRQGNSFDAGAATYNEPGRAYYVSMTTSF
\end{abstract}

\begin{abstract}
$>$ AbaBauA17978
MALRLGYALGTVFVLCASNTYATVIDNSTKTLEQQQAAQTNVAALPAITVKAEQDDTYAGGQVSSKSSVG FLGNKTAMETPFNTIAYTEKYIADQQAKDITDVIAKTDPSIFSTGAYGVIAESYAIRGFRSSSVAAGRDT SMGGLYGIAPLYRASPEMFERIDVLKGPSAMLNGMPPGSTLGGTVNLVPKRAGDDPLTRFTTTYMSDSQF GGHLDIGRRFGENKEFGVRFNSVYRDGEGPIKEQEKTTHLFSLGLDWRGDNARLSADLYTSRDHADGINR GLTILPGVNLPKAPNPDTLLNPTWTVYNTRDTGGMIRGEYDLNDKWMAYATAGMSKTEYNTLGAAKTEIQ NEAGDIKFNIAHLGFKYERKSAEVGLRGKFDTGAVKHALALNATHYRETDDEAGIRQGFPEGDRVTNIYN PNWGSKPNRVPIAPIFSNKENLTSVGLADTMSVLQDQVQLTLGLRYQNIVTESLNGQGVRNINGRYDKNA ITPAAAILVKANDHVSLYANYIEGLSSGGTVPTGQNYENEGQIFSPFKTKQMETGIKIDSGNFTNTFSVF QIKSRNSYVIKSTDEKIKSIYGADGEQRNRGVEWGFYGSPVDSIRVMGGLTYIDPKITKTRIASELGNVV AGVPKVQAKLGIEGDVSYIPGLTLTANATAASKQYIYNDNSTSVPGREVYGIGARYNTKIAQFPVTIRGN IDNLTNKSYWSMPQFTSLMLGAPRTYLLSATIDF
\end{abstract}

\begin{abstract}
>AbaBauA WP_001073039.1 ATCC 19606
MALRLGYALGTVFVLCASNTYAAVIDNSTKTLEQQTAQTNVAALPAITVKAEQDDTYAGGQVATSSNVGF LGSKKFLDTPFNTISYTDKYIEDKQAKDITEVIAATDPSIYTNGASGGWSENYYIRGYASSTNDMSMNGL FGITPFYRTSPEMFGRVEVLKGPSALLNGMPPAGSVGGTVNLVTKYAADEPFARLTTTYMSDAQFGGHVD VGRRFGENKEFGVRINGMYRDGDAAVNDQSKESRLFSLGLDWQGENARVFVDAYDALDHVDGVTRGVNVS TAVGIPKPPKADTLLSPDWGSVETKDKGAMIRGEYDFSDQLMAYAAYGQSTTEYKYNGASAGTITSSTGT LSSTLGQLAFDVDKKSADAGFKGKFETGSVKHQWVANATYYNHTQDDYGYRI IPGFSDPVITNIYDPNPN WGPKPEFTPPFLFHSTLSTSSFGLADTLSFAQDKVQLTLGLRHQTVKATSSVNTLPENAKSATTPGVALL IKATDKISVYANYIEGLTKGDQAPATASNPGEIFPPQKTKQQELGLKVDLGTFAHTLSAFEITKPSSYLD PSKLVNNLPTFVSDGEQRNRGIEWSFFGSPIEHVRLMGGFTYLDPELTKTKSGGNDGHTAVAVPKNQAKL GAEWDTQVAQGTLTLSGNINAVSKQYINAENTLSVPGRTLLDVGARYSTKVEDHPVTFRANIYNLTNKAY WAQPQLTNLALGAPRTYMLSVSYDF
\end{abstract}

\begin{abstract}
>AbaFhuA
MKRSVLFISSVGVMTSHVYALEADQQVVTNLPTISVKADKESSLKQEVGQASSATKGLMQLKDVPQIVNV VPKQVLREQTVTSMQGALQNVAGLSFSVGDGQRDQVMIRGFSAITDNYVDGIRDDALYFRDMSNVERIEV LKGPASVLYGRGSAGGLVNKINKKPMDQSLREVSLIGSTTGQRRAEVDVNEKVAENVKVRLTGAVEDSDG YRDQAFLKRQAVAPSVQWDITDKTKLLLQADYLHDNRLADQGFPTDPITGKPVKTNPKTFYGALNGKEVG DVDTEISSQTISLDHEFNDNFKYHGAVRHYNYSLDRQYSVDSHQKLPADQIQLTQNKRLRNEDGVYVQQE LSAVFNTGFLKHSTLIGAEYSKQHKDELVWSKARQITNIFNPQLENWAPLDTNVDADTNNTNTFENYGVY LQDLMTVTDQLKVLVGLRYDNLSQDRDDKTSKNVDLNRTDNTYSPRIGVVYQPVNNLSLYTSYNRSFQPL ADSFVFYKNSDDLRPTKTENYEIGAKWDVNDQLNVTLALFEMSQTNIQNKDPNDPKGLTAILAGEQKTKG
\end{abstract}


VEISLAGQLTDQLSVLAGYSYMDGKIEKSAIGFTGNHSALTPNNTANLWLKYQINDHWYAAVGGRGESSR FSAPDNKNVLPGYAVVNAALGYQSERYDVNLNLNNLFDRDYFVSGHSGANDSNMMGDPLNAQVALRYRF

\begin{abstract}
$>$ AbaFbsN
MLGDKQDLDIPFSITSYTSKYIADQQATSVADALRSEPSVRSVFSKGGIGEYFNIRGLYTQSHELAWNGL FGLLPHNRVPTEFLERVDVFKGTSALLNGMSIGGAVGGVINVVPKRATSEDITRLTTTYKSDSTFGAHID VGRRFGQEDQFGVRVNALKSLGDKALDGQEENRYLGSAAFDYKGEKFRASLDLYDINEKLDGGQPLMVSF ATSVVPKAPDSKLNHQPGSYSHTKTRGAIAHVEYDFAPEWTAFASAGTKRQNGVGVIANNALGNAAQPNG DYMAVSRMNANNTNVDAGELGVRGKFNTGAIKHNVVISANSLDQDTHMGLVMGTPWASNIYRPTRPALIA SPPTYTPKTLDTTLTSFAFADTLSILDDKYQLTLGVRHQNVKSQSYSILTGLPSSPYYDESAVTPMVGFV VKPWDNTLSLYANYIEGLSQGGTIIDPSNPLVAEKTFKPYKSKQYEIGTKWDMGKFKNTLSIYQITKNDL AQDANRNYSESEQRNRGIEWTTAGEIVKDIRLLGGVSYLEAEWKKTAGGLMDGNDVKGIPHWQSNLGLEW DVQPVEGLTLTANTTYTGSLYANDTNTQKIPDWFVLDLGGRYATNIAKHNVVFRGGVDNVFDKKHWAGGW NGYVGVGGDPRSYKLSMQIDF
\end{abstract}

\begin{abstract}
$>$ AbaBtuB
MEHVMSKSFQPTRLVGAIAIAMGCSPVIFAEDATDATQLDPIVITASKSAEKASEVPARISVISKEEIEK NPALNLSDILQKDASIYVKQNGGIGQLADLSLRGGQTGQTLLLKDGARLNSQNGFGPVNPEFINLTDVDQ IEILKGPASVQYGSDAISGVINLISKKPTKSGINLTGIYGENNTYKSIINADYVDASGFYAQVGGQRLES DGTRILSIQDKNDKAGFDQKGYHAKIGYQQNQIDTSISISENQGTNVYYDSTLNSNTGIRDFKNQLINWL GSYQVNPDIKLNARYSNIKNTRVSTDTDWQTYKLTSSHYNTETNESDLNAVWKFMEHQNILAGVTYSKSE YDASSTPEAIRSTNSTGYYLQHQYQKNGLNTQTGIRLEDNDWFGTHTVGQIAVRYQLLPLTSIYTNVGTA FKAPTLEHLYGSYGSSFYSNPNLKPEKSVSYEIGLDQKLNYGLSAYISGYSTDVENLISSGKIDGKTTYI NLNKAKIQGGEIGLKWVLDNLFANAEYVYSKAQNKETKLDTPYRPRQTYTLTVGYDDGLYGVNAAVVARS KANTSSANIKVPGYATVDLNAFWNISSNVKLFTNIQNIGDVENIVVNDFGSEYINGGRQASVGVTFRY
\end{abstract}

\begin{abstract}
$>$ AbaLGP1
MKKKYNVRSQATPKIGKTILKLSTLSLSMMCLTMAQAAETEQSSTDEKPTKVVKVAVTGSSIKGVAAQSA SPITIIKGEDLANAGVTTVEEALTRVSSNQAGFSTDQNVGASNTDGSTANLRGLGSDKTLVLLNGRRLAA NPFGTSTVNLNIIPLAMIDRIEVLRDGASAVYGADAVAGVINFITKKTYQGVGISAGLLQPEHKGGDKQD ISIFGGYGDLDEDGFNIFGVVDYRRTNGIMAKDRRISERGGVLPELGLDGRSANTFTSNFYDPISGVSGN PYAQNGQCLGPAESAEEGFCYANTQALIGIKPDVENVSVLGRGTFKVNDNLNAIGEYIYSRSEVITSVAP DPFSRSNLSTRVTLPSDSPYYPGNGIVPSVKGLSGAPLELYLRSHAGNRIAKSINESHRLFAGLEGDVWG WDLNTGVTYAKSEASDNFVTGQLNKTKLQDALNNGTLNPFGESSDPNIWNNLSVKGKYNEATLESTTADF SISRPIFTLPAGEVGFALGGSFTNQDWKQHINADLVRQAPSSGTDPSKPDNSGDRDITSAFAELQIPITK TIEAQLAARYDDYSDFGDTFNPKFAIRWEPLKQLMFRTSYSTGFRAPSLYEINAAQSKTYTGAKYNDPLY CPGGEVVEGKNKNDFCNTQFMKLQGGSKDLQPEESKSFTAGFVFEPIKNLVLTVDYFNIKIDDLIAQVGE ATIFGEPEKYSALFVRNADGTIDYIKTNLFNSGGMKTEGFDISLNYLTPMTSTGRFGFGIDGTYVSKLDY RDSKGEPWTGQVGLYEDPAVVRWKHTANINWAYENWKLVFEQQFVRGYDDQNQIGEEQYDHHKVSDYTLY NISGTYKGFKNLELTAGIKNIFDEDPSASNVLDNFQYGYDPRYGDPTGRTYYLRGTYKF
\end{abstract}

\begin{abstract}
$>$ AbaLGP2
MLNKSKLFLALITLGASKILLAAEGPVTTLNTIVLTAQSDELGSELLGKSLNVSNQFIDTSKLKQRSTTL GDALGTELGIHSNQYGGGASAPI IRGQEGKRIKVLQNNADVLDMSNMSPDHAVTVEPSLAKSIEIIRGAS TLLYSSNSAAGVVNVIDYKIPTQMPQDGLEGNTTLRFNTGSNEKLTTAGVTVGLSPRVALRAEGLYRNAG NYKTPHYQSSSYNSLEDLENQNIVYKNLKYLPESWAESRLGTLGLSWIDDNTYLGVSYTHRHDEYGLPAH SHLYEGCGASAISINTRISGLKNYLLYYPQLMEEQDINYVNPRPDCHQHNHIHETTFSHNAPYIDLNTRR YDMRGEFTQPFTGIDKIRTSLSYIDYFHNELEGDKITNFFKNTGKVGRIELSHQPLGELTGILGLQYLEQ DNSALSPVHSQEGHTTYLDTQQLLNRNVTKNFSVFGLEKYNWNDFTFELGARIEKQKVSMDYDIEKIKDS MKPWPNKYNSPYVEKNNKIRAQNLKSILEAVQPNKETAFSYAGTVHWRFAPNYILSLTGTHQERLPNAQE MYTHGMHLATNSFEIGNRFLRKEKSNNLEISLAYKDDLLDYQISTYYYDFDNYIYLQTLNEVLGTTKVRD QHTLRINHYSQSAANFYGLEGNIGYQFNSVYHGSLFGDYVKGRLTNLPDAVIAYDIWNREPTLAPQKDRY TPRLPPARLGTRLKADFDESLKGEIEYYRVFKQDNISKFEQVTSGYNMLNMTLAYKNKLSHTEYDLFFKA NNLLDQKVYAHETFLPYIPQIGRNFSLGLNLNF
\end{abstract}

\title{
$>$ AbaLGP3
}

MKRLFPLSLLSLMILNIQGANAEELIDSSSKAAATMPTIKIEAMSELDPIKSYIDYDKANVTRNGLDKKD IPQTVDTIDVQKYKIYGSNDLSVMLQGTPGVSTSYDMRGDGITIRGFGADTGDIYRDGIRESGQVRRSTA NIERIEILKGPASVLYGRSAGGGVVNMVSKFANFDSKSSVGAYAGSYDNYGTTADINQVLNDNLAVRLTG EYSEAGSFRSGIENKIEMFSPSFTYKNDDGKLTWTTQYTYDKLGRVPDRGPTRNNLPAGTSIKMGFAQDG DYVDDILQVVRTDVNYEYAPDWNFHWAASYRQAEQNFDHFYFGNYCGLDGKNSKNEACTKKGYIDQIYYW QQTSNKTTTNTFDIKGKFKTGQLEHQIMVGTDWTYEQREPRLANKTQNGSAIYGYVNPITGEREYSRGNG 
PLKISQHNYNEGTTYGVFMQDLIGLNDQLKLMMGLRYDYFDFSTTNKIKNEHRNVKDSTFSPNVGLVWQP VPEHSFYTSYSKSFAPFGGQMGVNQVTGSTDVAKMDKEPQYNEQYEVGVKSEWFDNRLNTQFSVFDIRKN NIRYKPNPDSEPEVWATAGQHQSRGLEFSFIGRVLDNVFVRGGYGYTDAKVKEDKQNPEREGNYLANTSK NTGNLFVRYLPTEQWYTEVGVTYVGSYYPNINNQVKMEGFNRVDAAIGYSADPWNVTLAVNNLTNKEYWR SDSMPGTPRNVLLRLNYQF

\title{
$>$ AbaLGP4
}

MRHKLGLLSSLGLSFISTLTWGEETSTVLETIRVQAESTREDVSQNSSATKFTHDVLDVPFNRAYLSKQI MEQQDVQRIDDALTLVSGVFHQNSFGGGFWDNYSFRGFSTDPNLGAAMIRNGLSVNRGINAPKDVVNIES LEFLKGPMAALYGRGETGGLLNLNSKKPQWESESELNLRANTQEQYRISLEHTAPINDELAYRLAVAHED NQSFRDHVSSERWFFSPQLTWKISDQTQLDFDSEFTEHKGTFDRGVSTVNHQFVMDPKTFTGEPDDGDLK IKDYFYQLRLSHEFNPDWKLNSAVSYKDAQMVGFATEPRRMQNDGRTLERQRRYRDYTSEDVLAQTELLG KLDTSWARHEILLSTELGQLDYKQNQLRRNHSTDSPNTIDIYQPEYGKYLPNLTPFTDTKERQRYFALNV QDQIFFNDQWSVLLGNRFDQVEQDFKNHIKQTEDNQTLHQNSPRFGVNFKASEQWAFYTNYGRSFAMNSG MNRNGQTFAPEKGESYEVGTKYKINDQSVLSLALFKMKKRNVLTTDPIDSNFQTAAGEVSSKGIEFDLNS QINDRWFVNANYSYTDAQIEKDQDLAKGARLSNVPKHQGAISTNYEFLQDGARKAGVGANLTYVGERSGH NLDNGFNLPSYTLVNLNGYYAPSDRLRYQLNINNLFDKTYYVSSYSDLWVQPGEPLNASISAQWKF

\begin{abstract}
$>$ AbaLGP5
MDLKLRLLSLSIAQLCCIPATFADTNNSSTTLATIKIKAQQASDQTYKVDSSSSATRSEIALQDTPQSVS VVTQKVIEDIGATRLVEALDLAGGVTRANNFGGQGLTGFNVRGFTSGEFYRNGFPINRGYPNAPDSNTIE RVDVLRGPSSSLYGRGDPGGTFNLISKTPNSEQQTTLGAQLNSEGLYRTTVDTTGTIPNAENIGYRLNVI AEGGDSYRDHVESKRYGIAPVIQWQATDATKVTFEADILRNQHPLDRGHTRYPTQKSFNSSPETYLWETG KYYNRLYNDNNMTQLRVEHDLGNDWKLNAGVQYLNGKLHGYAVEANGIQNDGETLGRNYNYRELKWQDTD AQINLTGNFQLLGLAHTLVTGLEYENYDYKSYI IRSSEDIGSYSINIYNPVLGQPLPELNTVTTHDRENL KTTALFVQDQLELNERLSALLGLRFEHYEHDYQDLRPGKPNWNTSHDAFIPRLGLVYKASDDLSLYGNAA KSFKPNTGASHSGEGFDPEEGMAYELGFKWLALNNMLSVDSAIFYANKENVLTNDPLFPNYKVAAGEVRS RGIELNIAGQITPAWKIIGGYAYTDAEVTKDNTLQKGTALANIPKNSFNLLNIYEFQDGPLQGLGLGINQ KYIDKRAGQTANSTYIMKGYAVTDLVSYYQATPKLRLNLDVKNIFDKVYDESAFNLYAYPGESRTVQLGM SYTF
\end{abstract}

\section{$>$ AbaLGP6}

MLFYKNILTLSILAAISIPVFAAENENVEKLETIRIKAHPLEQTSKDFAVADTVVDQKHLTEGAATIGDA LNSEVGIYANQFGAGSSRPVIRGQDGPRVKVLQNSSENVDVSTLSPDHAVTVDPVLAKQVEVIRGPSTLL FGAGTVGGLVNVIDNKIPTQMPENGYEGQVGLRYNTGSDEKLASAGVTVGLGSQVALRVEGLTRDANNYI APNYIHEGEKERRVDNTFAQGDSVNVGLSWIYDRGYTGISYSNRRDQYGLPGHSHEYETCSAHLTGRPHL HCDAHEHDHEEGEEAHAHEEHEHEHGGPWIDLKSERYDFKTELNDPFAGFQKLRAQASYTDYQHDEIEEG AIATRFQNKGYDGRIELVHNPIADWEGVIGTQLGQQKLNLTGEEAFMAPTTTKKWSVFALEHKQWKAVHF ELSARADQQEIDVDDNSKQDFDGSAFSYAGAANWEFAPNYKLSFVASHQERLPLAQELYANGAHFATNTY ELGNDQLSKEKSNNVELGLHFDNDKLDYHLHVYHNWFDDYIYAQTLDRYKDFRLVQYTQDKARFYGAEGE IGYQITPMYKISAFGDYVRGKIDAEGNAPRIPAGRLGTKVDADFGDGFSGSAEYYHIFNQDKIAAYETET EGYNMLNLGVAYSGQYGAKTDYRVYLKANNLLDDTVYQHASFLSNIPQVGRNFTVGVDFSF

\footnotetext{
PaeHXuA

MNKCYALVWNVSQGCWNVVSEGSRRRGKPAGAKAAIASVLALLGATALAPAYALPSGGTVVGGSANGEIH LSGGNSLSVNQKVDKLIANWDSFSVAAGERVIFNQPSSSSIALNRVIGTKASDIQGRIDANGQVFLVNPN GVLFGRGAQVDVGGLVASTLDITDAEFNGNSSRYRFTGPSTNGVLNQGGAITAAEGGSIALLGAQVDNRG TVLAQMGGVGLGAGSDLTLNFDGNKLLDIRVDAGVANALASNGGLLKADGGRVLMAARTANALLNTVVNS QGAIEARSLRGKNGRIVLDGGPDGKVMVGGALSANALNGPGHGGTVEVRGQAVEVALGTQVNTLASNGLN GTWKIAADKIDVRPSAVSDGVTVHADTLSRNLASTNIELVSTKGDLDLDGSVSWASGNRLGLGSAADLTL NGRLNASGAKAGLELKAEGAIDINDKIVLGGAGSALAMDAGEGHRVNGTASVSLAGANATYVSGGYYYTV VQNLAQLQAINKNLDGLYVLGGNILGGSYYCTALQSIGGPAGVFSGTLDGLGNSIGNLSISNTGPNVGLF ARSSGTLSNLKLNNLRVSDNTYGSGPSSLGALVGINSGRIANVSASGVSVVGSRLRSNALGGLVGRNING QITNASVSGGVTAYAASTAVGGLVGENFTTAWGPEAVIENAHSNVHVAAQSTERNSLGGVGGLVGLNAKA TIRASGSQGKVETYRPGLNVGGLVGYNMFGHVSDSSASGQVEAGGAGYTGGLVGLSSGGEIFRSQASGSV YSKGGLATGGLIGKAEGNGMLGNLKASGSVMDQGGADLGGLVGNNSQGAIETAEATGKVSGGSNSRVGGL IGRNLGGSVAHAISRGDVSGGFNSLVGGLVGHNGGELFNVDASGRVSAAASASVGGLVGSNAGSILSARS SSTVSGGGRSRIGGLVGENQIQGRIVSSMSEGTVSGDYYVSMGGLAGVNLGSIKYSGVSGKIDFKPQSHY GQIYGAQVGENRGVLGGNYVIGEAALLPPAGIDYGNIW

$>$ PaeHasR

MKHRGWSAVRGGRKGAQLALGLGLVLLGTAALPLHAQDGADSASQQQTALRRVRLDIPAQPLNRALLRFA
} 
EQAGVQVFFDSQRFAGLGSAAVHGEYLLADGLSQMLQGSPVEYRFSGKDQLSLIRVSQDDLVQMSPSVIS AARPDDWVYQTPHSVSVIGREQIERNPPRHAADMLEETPGVYSSVSQQDPGLSVNIRGIQDYGRVNMSVD GMRQNYQQSGHQQRNGTLYVDPELLSEVVIDKGASSAMGGAGVIGGIANFRTLEARDLVRPGKQVGGRVR LTSGLGGDANGTHFIGSAAFAIGTEVWDMLVAASERHLGDYDPGTKGSIGELRTGAWFNPEAGQRVKHSP VAYSGYVMRSRLAKLGVALPQDQRLQFSYLTTQVSYDDANMLNTENQALWEKLGSSDVRAQNFAIDYGYA PDNPLVDFKAKLYYVDNRNRQQTLQRGITPGYSITYQTDTYGAQAQNTSTFALDDLSTLRANYGLEFFYD KVRPDSSQPRASTSAVGFPAAEGMTPKGDRALGSLFARLDYDYDDWLNLNAGLRYDRYRLRGDTGFNART FILGTTRQTDMPLQYAVDREEGRFSPTFGLSVKPGVDWLQLFATYGKGWRPPAVTESLITGRPHGGGAEN MYPNPFLSPERSKAWEVGFNVLKENLWFSDDRLGLKVAYFDTRVDDFIFMGMGMQPPGYGMAGIGNSAYV NNLDSTRFRGVEYQLDYDAGLAYGQLSYTHMIGSNDFCSKTAWLGGVTQTVKGSGRRPPVIDMRPDEQAN AATHCSAVLGSAEHMPMDRGSLTLGMRFFDRRLDVGARARYSEGYSVAGGATVSQAGVYPADWKEYTVYD LYGSYRVSDELTLRLAMENVTDRAYLVPLGDVLAFTLGRGRTLQGTLEYQF

\section{>PaeFpvA}

MPAPHGLSPLSKAFLMRRAFQRRILPHSLAMALSLPLAGYVQAQEVEFDIPPQALGSALQEFGRQADIQV LYRPEEVRNKRSSAIKGKLEPNQAITELLRGTGASVDFQGNAITISVAEAADSSVDLGATMITSNQLGTI TEDSGSYTPGTIATATRLVLTPRETPQSITVVTRQNMDDFGLNNIDDVMRHTPGITVSAYDTDRNNYYAR GFSINNFQYDGIPSTARNVGYSAGNTLSDMAIYDRVEVLKGATGLLTGAGSLGATINLIRKKPTHEFKGH VELGAGSWDNYRSELDVSGPLTESGNVRGRAVAAYQDKHSFMDHYERKTSVYYGILEFDLNPDTMLTVGA DYQDNDPKGSGWSGSFPLFDSQGNRNDVSRSFNNGAKWSSWEQYTRTVFANLEHNFANGWVGKVQLDHKI NGYHAPLGAIMGDWPAPDNSAKIVAQKYTGETKSNSLDIYLTGPFQFLGREHELVVGTSASFSHWEGKSY WNLRNYDNTTDDFINWDGDIGKPDWGTPSQYIDDKTRQLGSYMTARFNVTDDLNLFLGGRVVDYRVTGLN PTIRESGRFIPYVGAVYDLNDTYSVYASYTDIFMPQDSWYRDSSNKLLEPDEGQNYEIGIKGEYLDGRLN TSLAYFEIHEENRAEEDALYNSKPTNPAITYAYKGIKAKTKGYEAEISGELAPGWQVQAGYTHKIIRDDS GKKVSTWEPQDQLSLYTSYKFKGALDKLTVGGGARWQGKSWQMVYNNPRSRWEKFSQEDYWLVDLMARYQ ITDKLSASVNVNNVFDKTYYTNIGFYTSASYGDPRNLMFSTRWDF

\section{$>$ PaePupA}

MPTAHAVSPLARALGSLGANKMSRLGLALLLVSAQAVA QEYEFNVPAQRLDAALQALGRQAGLQVLYNPS DLSGLRSRSVSGKLELEQAISQLLDGANGIGFELQGKTLILRLQDSSSVISLDSMVVSGLALNPTTENSG SYASPAVSIGKGNKSIKEIPQSVTVVTQKRMQDQNMSTVSDVLANAPGVTSIPMFGTGEQYYSRGFFIEN FQYDGVPLERQSYARGSDFSGQTAIFDRVEILRGAQGLLEGGGNPSGSVNFVRKRPTAENQVRLTAKAGS WDHYGTQADISGPIDEQGRLRGRLVLDYDTKNSFVDHVGDDRQTIYAALDYDLTEATRLGFGFSRERIDA NIDVNGLPNYTDGSMPHFSRSTFLGGDWSYWDKIQETFYFDIAHQFNEDWSLNATVVNAREKNDYKYLLM AGSINPDNTSTLRGDAYVFDFFSEHWGGDVYVNGRTKVADRQLNLTFGANYSDLDSSDTFGWRQRYVPNW DIFNSPVNTNKPSDDDIYSVRRSDDGYASIQKGVYGMGQYYLTDDLSVVLGTRISSYQKAYTSNGPWGYS KAVAKESAKAIPYAGI IYDLNPEWSAYASFTKIFLPQSVRTASGSILGPKSGKSVETGLKGILNDGRLNA TFALFRMEQDNIPIQDANLPDDVQEANCGGTCYHGGGKAISRGFETELSGELFDNVQVYAAYTLNLLRYQ GEVPSEADDVGASVNTPKHIFRSWVDYRLPGNWSRVSVGGGVNAQSRSAGFGYYGREQSGFAVWNSRLAY RFTEELTGAMNVNNIFDRRYFKSVDYGHNYFGDPRNVLFTLTYSY

\section{$>$ PaeFoXA}

MTATAVVLRNAPSSLDFPRASRLSRSVRAALLSLAMAAGAAPLCASAAEAAAEQARPYAIPAGQLGDVLN RFAREAGITLSATPAQTGGYSSQGLRGSFTVQQGLARLLADTPLEAEDQGDGSFVLREAPAKDGDVLNMQ AVEVFALGNNLGSTDGYLATHSQIATKTSKPLLETSQTVSVITREQIDDTASKTVQQAMRYTPGIFTGQV GASNRYDYVVMRGFADNSVDNIYLDGLKAMGDSGTFSSMQVDPYFLERIDVLKGPSSVLYGRSLPGGLVA LTSKKPLYEDYRQITGSIGNMGQKEMGFDFSGPLDEEKRIAYRLIGLGKGSDTQFDHVKEERYAIAPTLA IDFSDDTTLTLQGYLQHDPNGGYHGGVPADGTLSHHNGRHISREFFDGEPSKDDFDRTQRMFGYQLEHRI DDVWSARQNFRYLDSDVDLSQVYAYGWSASEPNKLNRYFSGAREHLQAYIVDNMLQAEFATGAARHTLLT GLDYQRRRTVVDWRSGSASALDAFNPVYGDDAISYFPDDNHTRRLEQTGVYLQDLIDIDQWRFSLGLRQD WVSVTDKNRSTGSKADDDWEKFTGRIGALYLFDNGLAPYVSYSESFNPNAYSDASGTPLAPTEGKQWELG LKFQAPGSNSFYTASLFHITQENVASKEPQDNFYTSVGEVRSQGLELEAHTQLSDNLKLLGSYTYTDITY TKSLDGNQGHTPNQAPKHMASLWADYAFDAGPLSGLSIGGGARYVGETWADKENTLRVPDYTLVDARIGY DLGKLGLKGLDVSLNANNLLDKDYVASCYSLDFCYFGEKRNVTATVNYQF

\section{$>$ PaeChuA}

MPLSPPFALRPCLALLLLSPSLALAGNAVPLTPTTITATRTEQAVDSVPSTVSVQTREQLDRQNVNNIKE LVRYEPGVSVGGAGQRAGITGYNIRGIDGNRILTQIDGVELPNDFFSGPYAQTHRNYVDPDIVKRVEILR GPASALYGSNAIGGAVSYFTLDPSDIIKDGKDVGARLKAGYESASHSWLTSATVGGRADDFDGLLHYGYR QGHETESNGGHGGTGLSRSEANPEDADSYSLLGKLGWNYAEGSRFGLVFEKYKSDVDTDQKSAYGGPYDK GKPAIPPSMLPGGMYQWRKGNDALTRERYGLEHHFLLDSQVADRIQWSLNYQLAKTDQATREFYYPITRK VLRTRDTTYKERLWVFDSQLDKSFAIGETEHLLSYGINLKHQKVTGMRSGTGTNLDTGADSPRDALERSS 
DFPDPTVKTYALFAQDSISWNDWTFTPGLRYDYTRMEPHITDEFLRTMKQSQNTAVDESDKKWHRVSPKF GVTYDFAQHYTWYGQYAQGFRTPTAKALYGRFENLQAGYHIEPNPNLKPEKSQSFETGLRGKFDEGSFGV AVFYNKYRDFIDEDALNTDSTGGNGQTFQSNNIERAVIKGVELKGRLELGAFGAPQGLYTQGSVAYAYGR NKDNGEPINSVNPLTGVFGLGYDEADGNYGGLLSWTLVKRKDRVDDSTFHAPDGTASQFKTPGFGVLDLS AYYRLSKDLTLNAGLYNLTDKKYWLWDDVRGYDSVGEASALAPANIDRLSQPGRNFAVNLVWDI

\title{
>PaeFepA1
}

MNNRIKSLALLVNLGIYGVAFPLSAAETATDDKNSAAEETMVVTAAEQNLQAPGVSTITADEIRKRPPAR DVSEIIRTMPGVNLTGNSTSGQRGNNRQIDIRGMGPENTLILIDGKPVTSRNSVRLGWRGERDTRGDTSW VPPEMIERIEVIRGPAAARYGNGAAGGVVNIITKKTGDEWHGSWNTYMNAPEHKDEGSTKRTNFSLSGPL GGDFSFRLFGNLDKTQADAWDINQGHQSERTGIYADTLPAGREGVQNKNIDGLVRWEFAPMQSLEFEAGY SRQGNLYAGDTQNTNSNDLVKENYGKETNRLYRNTYSVTWNGAWDNGVTTSNWAQYERTRNSRKGEGLAG GTEGIFNSNQFTDIDLADVMLHSEVSIPFDYLVNQNLTLGSEWNQQRMKDNASNTQALSGGTIPGYDSTG RSPYSQAEIFSLFAENNMELTDTTMLTPALRFDHHSIVGNNWSPSLNLSQGLWDDFTLKMGIARAYKAPS LYQTNPNYILYSKGQGCYASKDGCYLQGNEDLKAETSINKEIGLEFKRDGWLAGVTWFRNDYRNKIEAGY APVYQNNKGTDLYQWENVPKAVVEGLEGTLNVPVSETVNWTNNITYMLQSKNKETGDRLSI IPEYTLNST LSWQVRDDVSLQSTFTWYGKQEPKKYNYKGQPVTGSEKNEVSPYSILGLSATWDVTKYVSLTGGVDNVFD KRHWRAGNAQTTGGATGTMYGAGAETYNESGRTWYMSVNTHF

\begin{abstract}
>PaePiuD
MSRQSTDTAVSSPRLLASAIGVAITAIAAPQAAHA DEAGQKKTDKDRVLSLDAATIVGEQQDETTYNVDR SASKKYTAPLLDTPRSVTVVPKQVIKDTAAVSLQDALRTVPGITFGAGEGGNPTGDRPFIRGFDAQSDTY VDGVRDTGAQTREIFNLEQIEVSKGPNSAFGGRGSAGGSLNLVSKQAKAGNFIDGGFTYGSDQTRRYTLD LNQEFLDGNAAFRLNLLKHDANVAGRDEVDVSRWGVAPSLTFGLGSPTRVTVSHYHLESDDTPDSGIPYA KSSDRSKHNPDKPVNVDRGNFYGLTGRDFQKSRIDTSTITVEHDLTDSLTIRNTSRYGNSHQDYLWTQPD DSQGNINNGSVWRRQNNRVSTTTTAVNQTDLFGEFYLGGFKNSFSTGLEFSREDSKRDGYIVDTNTGLGS NKCNPSLIGAPSGYNCTSLENPNPHDPWNGSITRKYAPLNTVGTTKAIYAFDTIDLNEQWQVNIGARFDS FETTAKNHGVRPATKLSDKSSFWNWQAGLVWKPVPNGSIYASYATSATPPGSMLDNGDTSNAVDGFAISN NLEPEETTNYELGTKWAFFNERLELSAAIFRTDKDNTRILVANQTYDNAGQSRVDGVELSASGKLTEKWK VFAGYSYLDSELVDAGKAGRNGNVNASAASNNGNEMPNTPKNSFSLWTTYDIFPKTTIGGGAFYVDKVYG DVGNTVYVPDYWRYDAMASYKLSKNVDFQLNVQNVFDKKYFDKAYAAHYASQAAGRTILFSTNFHF
\end{abstract}

\section{$>$ PaeFepA2}

MKYTSHFPLGIVIPLLA CSVPLQAAENMTEQSTRDKSTPTAENQEETMVVTAARQNLQAPGVSTITAEEI RKHPPARDVSELIRTQPGVNLTGNSTSGQRGNNRQIDIRGMGPENTLVLVDGKPVTSRNSVRYGWRGDRD SRGDTSWVPAEMIDHIDVIRGPAAARYGNGAMGGVVNIVTKPTTPEWHGSWNTYMNAPQHRKEGATKRTN FSLNGPLSDSVSFNLWGNLSKTQADAQDINAGHEAERTGSYAGSYPAGREGVVNKDIHSKLRWEFAPMQA LEFEAGYSRQGNLYAGDTQNTNTSTLVKSMYGKETNRLYRQTYGVTWTGGWDNGVTSNSYAQYEHTRNSR MNEGLAGGTEGIFSSSEFSDIDLADVLLHSEVNIPFTLGVDQNLTLGTEWNQQRMKDGVSTTQALSYGTI DGVSATGRSPYSSAEIFSLFTEDNMALTDSTMLTPALRFDHHSIVGNNWSPSLNLSQELTDDWTLKLGIA RAYKAPNLYQLNPNYILYSNGQGCYASSSACYLMGNSDLKAETSVNKEIGLEYKHDGYQAGITWFRNDYH NKIESGYAAVGTASNGTTNIYQWENVPKALVEGLEGTLNLPVGETVNWSNNLTWMLQSKNKTTGDRLSVI PQFTLNSTLSWQVREDLSLQSTFTWYGRQKPKRFNYKGEAVSGSELNEVSPYSIVGLSATWDVNKNLSFT SGIDNLFDIRHYRAGNAQTTGNATTGAYLYGAGAETYNESGRTFFMSVNTHF

\section{>PaePfeA}

MSSRALPAVPFLLLSSCLLANAVHAAGQGDGSVIELGEQTVVATAQEETKQAPGVSIITAEDIAKRPPSN DLSQIIRTMPGVNLTGNSSSGQRGNNRQIDIRGMGPENTLILVDGKPVSSRNSVRYGWRGERDSRGDTNW VPADQVERIEVIRGPAAARYGNGAAGGVVNIITKQAGAETHGNLSVYSNFPQHKAEGASERMSFGLNGPL TENLSYRVYGNIAKTDSDDWDINAGHESNRTGKQAGTLPAGREGVRNKDIDGLLSWRLTPEQTLEFEAGF SRQGNIYTGDTQNTNSNNYVKQMLGHETNRMYRETYSVTHRGEWDFGSSLAYLQYEKTRNSRINEGLAGG TEGIFDPNNAGFYTATLRDLTAHGEVNLPLHLGYEQTLTLGSEWTEQKLDDPSSNTQNTEEGGSIPGLAG KNRSSSSSARIFSLFAEDNIELMPGTMLTPGLRWDHHDIVGDNWSPSLNLSHALTERVTLKAGIARAYKA PNLYQLNPDYLLYSRGQGCYGQSTSCYLRGNDGLKAETSVNKELGIEYSHDGLVAGLTYFRNDYKNKIES GLSPVDHASGGKGDYANAAIYQWENVPKAVVEGLEGTLTLPLADGLKWSNNLTYMLQSKNKETGDVLSVT PRYTLNSMLDWQATDDLSLQATVTWYGKQKPKKYDYHGDRVTGSANDQLSPYAIAGLGGTYRLSKNLSLG AGVDNLFDKRLFRAGNAQGVVGIDGAGAATYNEPGRTFYTSLTASF

>PaePiuA

MEKNASLPFGSFNSLALFTGLCLGASPATGIAAEKSVKNSEETLVVEATPPSLYSPGASADPKFNKPLVD TTRTITVIPEQVIKDQGVTNLTDALKNVPGVGAFYAGENGSSTTGDAIYMRGVDTSNSIYVDGIRDIGSV TRDTFNTQQVEVIKGPAGTDYGRSAPSGSINMISKQPRLDSGIDGSASIGSAWSRRGTLDLNQAFSDSAA 
FRLNLMGEKTHDAGRDSIENERYGIAPSLAFGLDTPTRLYLNYLHVRQNNTPDGGIPTVGLPGYSAPSPK YAALNSAGKVDTSNFYGTDSDYDKSTTDSGTLRFEHDLTDNTTVRNTTRWSRVKQEYLLTAVMGGASNIT APDINDVNTWSWSRLVNTKDVSNRILTNQTNITSTFNTGSIGHDVSAGVEFTRENQTNYGVNAMTAPAVN LYHPVSNLSIGGLDRNGANANGQTDTFGIYAFDTLTLTERIEVNGGLRLDNYHTKYDSATACGGSGRGAI ACPPGQSTGSPVTTVDTAKSGNLVNWKAGALYRLTEQGNVYVNYAISQQPPGGSSFALAASGSGNSANRT DFKPQKAKSSELGTKWQIFDNRLLLSAALFRTDIENEVAANDDGTWSQYGKKRVEGYELSATGNLTPDWT IIAGYTQQHATVTEGQNVAQDGSSALAYTPKHAFTLWTQYQATSDLSVGGGVRYVGSLRRGSDGAVGTPD HTEGYWVADAKLGYRFNSNLDLQLNMYNLFDTDYVASINKSGYRYHPGEPRTFMLTANVHF

\begin{abstract}
>PaeChtA
MPRSIPSRPAPLALSLSLFASFSAPTLAADPVEQQMVVIGSRAPTSISELPGTVWVIEREQLDQQTQAGV PLKEALGQLIPGLDIGSQGRTNNGQNLRGRSVLVMIDGVSLNSSRGISRQFDSIDPFNIERIEVMSGASA VYGGGATGGI INIVTRKGVGGDTRFNTELGARSGFQSHEDHDLRAAQSISGGNDLFNGRLAIAYQKNGAA YDGNGDQVLTDITQTDLQYNRSVDLMGSLGFTFANGHSLDLGLQYYDSGYDGDRGLDLGRNFDALRGRAP YSIKGGVDLDREPESKRHQFNATYHAPEVLGHDLYLQAYYRNEKMAFNPFPTIRYNNSGAINYGTSYYSA SQQDTDYYGMKLALVKTWERVSLTYGVDLDREKFTSDQMLFNLPLAAASGGLVASEQAKLGRYPDIDTDS RAFFLQGSWKATDDLTLSAGVRRQSMSTDVSDFVAANQQILIANGLGKSADAVPGGSKDYDVNLVNVGAI YKLNQQQQVWANYSEGFELPDPAKYYGFGRYGAADGNGHYPLLQGVSVNDSPLDGIKTKQVELGWRHTDG ALDTQVAAFYSWSDKSIKYDSKTLAVLQQDTKKRNYGFEGQATYWLDDHWQVGVNGLAIRSQEKVDGRWL KQDVTSASPSKAGAFVGWKDDQRSLRLQGVRTFNLNDEAGNKIDGYALFDLLGTQALPVGSLTAGIQNLL DKDYTTVWGQRAQVYYGGLAPAGLFDYKGRGRTYSLTYSVEF
\end{abstract}

\title{
>PaeIutA
}

MKKRLWVLHPLLLVSSLPALAAQSDEDSIIVSANRTHRTVAEMAQTTWVIEGQEIEQQVQGGKEFKDVLA QLIPGIDVSSQGRTNYGMNMRGRAIVVLIDGVRLNSSRTDSRQLDAIDPFNIEHIEVISGATSLYGGGST GGLINIVTKKGQQDRQVDLEVGSKSGFANSNDHDERIAAAVSGGTDHASGRLSVAYQRFGGWYDGNNDAL ILDNTQTGLQHSDRLDVMGTGTIEIDDNRQLQLVTQYYKSQGDDDYGLWLGKNMSAVTSGGKAYTTDGLN SDRIPGTERHLISLQYSDADFFGQNLVSQVYYRDESLTFYPFPTLTKGQVSSFSSSQQDTDQYGAKLTLN SQPLAGWDLTWGLDADHETFNANQMFFDLQQSLASGGLHNESIYTTGRYPGYSISNVAPFLQSSYDLNEI FTVSGGVRYQWTENRVDDFVGYAQQQDIANGKARSADAIKGGKTDYDNFLFNAGIVAHLTERQQTWFNFS QGVELPDPGKYYGIGKYGAAVNGHLPLISSVNVDDSPLQGIKVNSYELGWRYTGDNLRTQLAAYYSTSDK TIVVNRTDMTIDVQSDKRRIYGVEGAVDYFIPDSDWSVGGNFNVLKSQVQTDGRWQKWDVTLASPSKATA WVGWAPDPWSLRVQSQQVFDLSDAAGNKLEGYNTVDFIGSYALPVGKLTFSIENLLNEDYVT IWGQRAPL LYSPTYGSSSLYEYKGRGRTFGLNYALTF

\section{>PaePirA}

MYPQFRRGHLAAAVLFASSSLLGGQALAEDERLEELDERAESVVQLGDEVVLGTAEQELKQAPGVSIITA EDIRKRPPVNDLSEIIRTMPGVNLTGNSSSGQRGNNRQIDIRGMGPENTLILVDGKPVSSRNSVRYGWRG ERDTRGDSNWVPPEEVERIEVLRGPAAARYGSGAAGGVVNIITKRPTDRLRGSMTVFTNIPESSKDGATR RANFSLSGPLTEALSFRAYGSANKTDSDDTDINLGHTVNPSRTVAGREGVRNRDLSGMLSWQVTPDQVVD FEAGFSRQGNIYAGDTQNNNGTANTQGLADDGAETNRMYRENYAITHNGTWSFGTSRFVAQYDSTRNNRL EEGLAGSVEGQIGADRSFSASKLENYRLSGELNLPLHALFEQVLTVGAEWNKETLNDPSSLKQGFVGSDS LPGTPAAGSRSPKSKAEIRALYVEDNIELRPGTMLTPGLRLDDHSDFGLNWSPSLNASQTLGEYFTVKAG IARAFKAPNLYQSNPNYLLYTRGNGCPIQTSSGGCYLVGNENLDAETSVNKELGIEFRRDGWVAGLTYFR NDYKNKIVAPLDVMGQTGTGNNILQWSNAKKAVVEGLEGNLLVPLHEDLSWSTNLTYMLQSKDKDTGNPL SVIPEYTLNSTLDWQASERLSTQLTSTIYGRQEPPKHGTSRNTPVVSRKEVGTYGIWGVSAGYTFSENLS VRGGVSNLFDKRLYRQGNSFDAGAATYNEPGRAYYVSMTTSF

\section{>PaeFhuA}

MARPKTAQPNHSLRKIAVVVATAVSGMSVYAQAAEQPKQEETITVVAAPAAQESAWGPAPTIAAKRSATA TKTDTPIEKTPQSVSVVTRQEMEMRQPTTVKEALSYTPSVFSTRGSSTTYDVVTIRGFTTSTTVNTNQYL DGMKLQGNNYSEVSMDPYFLERVEVMRGPTSVLYGNSNPGGIVSMVSKRPTTEPLKEVQFKMGTDNLWQT GFDFSDAIDDAGVWSYRLTGLGRSQDTQQQMAKSTRYAVAPSFSWRPDDKTDFTFLSNFQNDPDAGYYGW LPREGTVVPYYDANGKAHKLPTDFNEGEADNKISRRQKMVGYSFSHQFDDTFTVRQNLRYAEVDTLYRSV YGNGYVAPGYMNRAYVRSDEHLNTFTVDTQLQSAFATGAVSHTLLTGVDYSRMRNDVDADYGTADPISMS NPQYGNPNIQVTFPYAVLNRMEQTGLYAQDQMEWDKWVMTLGGRYDYATTSTLTRSSNSLAENHDQQFTW RGGVNYLFDNGISPYFSYSESFEPVSGSGLNGQPFDPSRGKQYEAGVKYVPKDMPVVVTAAVYQLTKDKN LTADPANQAFSIQTGEIRSRGLELEAKAAVNANINVTAAYSYTDAEYTHDTVFNGKRPAEVPRNMASLWA DYTFHETALSGLTIGAGARYIGSTVSYYKTDTSTGKKNDSFSVAGYALMDATVKYDLARFGLPGSSVGVN VNNLFDREYVSSCYSEYACYWGAGRQVVATATFRF

>PaeIroN 
MYPHFRRGHLAAAVLFASSGLLAGQALAEDERLEELDERSESVVQLGDEVVLGTAEQELKQAPGVSIITA EDIRKRPPVNDLSEIIRTMPGVNLTGNSSSGQRGNNRQIDIRGMGPENTLILVDGKPVSSRNSVRYGWRG ERDTRGDSNWVPPEEVERIEVLRGPAAARYGSGAAGGVVNIITKRPTDRLRGSMTVFTNIPESSKDGATR RANFSLSGPLTDALSFRAYGSANKTDADDTDINAGHSVDPTRTVAGREGVRNRDLSGMLSWQVTPDQIVD FEAGFSRQGNIYAGDTQNNNGNANTQGLADDGAETNRMYRENYAITHNGTWEFGTSKFVAQYDSTRNNRL EEGLAGSVEGQIGADRSFSTSKLENYRLNGELNLPLHALFEQVLTVGAEWNKETLNDPSSLKQGFVGGDS LPGTPAAGSRSPKSKAEIRALYVEDNIELRPGTMLTPGLRLDDHSDFGLNWSPSLNASQALGEYFTVKAG IARAFKAPNLYQSNPNYLLYTRGNGCPIQTSSGGCYLVGNENLDAETSVNKELGLEFRRDGWVAGITYFR NDYKNKIVAPLDVMGQTGSGNNILQWSNAKKAVVEGLEGNLLVPLQADLSWSTNLTYMLQSKDKDTGNPL SVIPEYTLNSTLDWQASERLSTQLTSTVYGRQEPPKHATSRGSRAISRKEVGTYSIWGVSAGYTFSENLS VRGGVSNLFDKRLYRQGNSFDAGAATYNEPGRAYYVSVTTSF

\section{>PaeFptA}

MKTETKVIKGRQGIARNRHTPLCLGLLLALSPLAAAVADARKDGETELPDMVISGESTSATQPPGVTTLG KVPLKPRELPQSASVIDHERLEQQNLFSLDEAMQQATGVTVQPFQLLTTAYYVRGFKVDSFELDGVPALL GNTASSPQDMAIYERVEILRGSNGLLHGTGNPAATVNLVRKRPQREFAASTTLSAGRWDRYRAEVDVGGP LSASGNVRGRAVAAYEDRDYFYDVADQGTRLLYGVTEFDLSPDTLLTVGAQYQHIDSITNMAGVPMAKDG SNLGLSRDTYLDVDWDRFKWDTYRAFGSLEQQLGGGWKGKVSAEYQEADSRLRYAGSFGAIDPQTGDGGQ LMGAAYKFKSIQRSLDANLNGPVRLFGLTHELLGGVTYAQGETRQDTARFLNLPNTPVNVYRWDPHGVPR PQIGQYTSPGTTTTTQKGLYALGRIKLAEPLTLVVGGRESWWDQDTPATRFKPGRQFTPYGGLIWDFARD WSWYVSYAEVYQPQADRQTWNSEPLSPVEGKTYETGIKGELADGRLNLSLAAFRIDLENNPQEDPDHPGP PNNPFYISGGKVRSQGFELEGTGYLTPYWSLSAGYTYTSTEYLKDSQNDSGTRYSTFTPRHLLRLWSNYD LPWQDRRWSVGGGLQAQSDYSVDYRGVSMRQGGYALVNMRLGYKIDEHWTAAVNVNNLFDRTYYQSLSNP NWNNRYGEPRSFNVSLRGAF

\section{>PaeFhuE}

MNRSAFRIPQPQRLSVAVLAALCAVAPAAFADTAADPTTLDKVVVKGERAEGYSVRRTSAGTRFDLAPRE IPQSLSIISHQRIEDQNLDDI IDVLANTTGVSSTQSDSERTEFYSRGFYIDSYQFDGLPTQMVQNWSYGD SGLDLALYDRVEVVRGATGLLTGAGNPSASVNLVRKHADSAELTGSVSVNVGSWGRTRSTVDVTTPLNKS GSVRARVIGSYLDTDAQMDRYNQHKTLGYAVIDADLTPDTQLSVGYDYQQKRANGATWGGFPMLFSDGTS TGYDESFNPNPSWTYWDTTSKRAFATLEHAFNDDWKVRIGATHDETKADDKLFYPAYNDWVTGASFFDKN TGAGISPSAGFYNTERKVNAVDGYFSGAFELFGLKHEVMGGLSYNKREYANYGDYQIGGAGLAWDPFSSY LNWNGNISEPNWNPLALASEGTITQKAAYAAARLSLADPLKLIVGARYTDWKSEGEGADRSHKVTSPYAG LVFDINDTYSTYASYTEIFQPQMLKDRNGSYLDPVDGKSYEVGVKGAWFDNRLNASVAVFRIEQDNVGQS TGEPVIGGTGGETAYVAARGTVSRGFEFELNGELAPGWNGTFGASRYVAKDINGADINTNLPQTALKLFT SYTPQSLQALTVGGGANWQNRIYYAVPAYGRIEQSGYALVSAFVRYRLSPEWSVQANLNNLLDKKYFSQI NGYGAYGDGRNGSLTFTWAF

\section{$>$ PaeFvbA}

MNARTHRTLNRGIGVVTLSTLLASPSALARENPPAEDEPAALEALVVTGEKRERSLKDTASSVSLTSARD IDRKQTGNASVAEVINGSPNVVYTDSVGAPIIRGQDTQGPNNGQNVFWGGTVPRATINLDGHYLNYNEMF FGATSVWDVDSIEVFRGPQTTSQGANAIAGAIIVNTKDPTFSPEAAYQAEIGSYHSRRSSIAVSGPLAQD FAGRLAVDYAGRDTFIDYDNPKFQDSGTDQDFRALNARAKLLWLPSGIPGLESKFTFSHNDSNRPTQEAA TRPFDKLDHRSTTMPSWEQDTNTSILDVAYDLDNGIRLFNQAQYSLSSVHRTTGAGGEGDADIRQKNASN ESRISFGEQEDRISGMGGVYYARTRTDETLHLRGLSAFDDTKKNLGVFGELNYRLSDRWTLTTGLRYQED RIERSGNSVLAPRPLDYQKTFSAFLPKVSLAFAATPDWTVGGLVSRGYNPGGVSLNLTTRNWAYFKEETI WNYELFTRASLLDGRLLLNGNLFFMDFKDAQYNIPVVVSPGVAQSYTINAEKAHAYGMELDLDYRLRDDL RLKASAGVLRTRIDEMSGNTGYEHNEFARSPGYTLSFGPSWDVTERLNLNAQVRYLDGYYSDTANTKAYS IKAYTLTDARASYRFNDQVQLYGYVKNVFDDRSPTYMQENRGIGGIEASMTQPRTLGIGIKGTF

\section{$>$ PaeCirA}

MFRLNPFIRAGLSASVVSLAFPALADVNEETLVVTASATEQSVKDAPASISVITQQDLQRKPVQNLKDVL RDVPGVQLTNEGDNRKGVSIRGLGSSYTLILVDGKRVNSRNAVFRHNDFDLNWIPVDAIERIEVVRGPMS SLYGSDALGGVVNIITKKIGQKWTGTLSADTTIQEHRDRGDTWNGQFFTSGPLIDGVLGMKAYGSLAKRA KDDPQSSSSATGETPRIEGFTSRDGNVEFAWTPGENHDFTAGYGFDRQDRDSDSLDRNRLERENYSLSHN GRWDMGNSELKFYGEKVDNKNPGQNGTITSESNAIDGKYVLPLDMINQLVTFGGEWRHDKLKDPVNLSSG GQSTSASQYALFIEDEWRIIEPLALTTGIRMDDHQTYGDHWSPRAYLVYNATDTVTVKGGWATAFKAPSL LQLNPDWTTNSCRGSCSIIGNPDLKPETSESFELGLYYRGEEGWLQDVEGSITTFQNNVDDMIDVLRTSS ASEAPGYPNFVGWKTVNGKRVPIFRYFNVNKARIKGVETEVKIPFGDEWKLTMNYTYNDGRDLSNGGDKP LQTLPFHTANGTLDWKPLDDWSFYVTANYTGQQRAVSATGKTPGGYTLFDVGAAWQVTKNVKLRSGVQNV GDKDLSRDDYSYTEEGRRYFMAVDYRF 
$>$ PaeBtuB

MIKKASLMTALSVTAFSGWAQDSNSDTLVVTANRFQQPVNTVLAPTDIVTRDDIDRWQSKDLNDVMRRLP GVDIARNGGMGQSASLYVRGTEARHVLVLIDGVPMARPGISNGVDISQIPISLVQRVEYIRGPRSAVYGS GAIGGVVNIITMTDSDRSQVNVGMGSNGYQTYDGAINKRFGDTVVTAAGAYQTTKGFNVQPNSPYSGDSD RDGYRNKLFLGGVQHKFDDNFSGFFRGYGYTANTDYDQGSYGYVGGNDERQNYTQSWDTGLRYSSGIYSS QLIANYQRIKDYNYSSDAGRYAAGATLDDMEQRYIQWGNNIEVGHGAISGGVDWKQEKLTSSSTTLSDAY KRDTTGLYLTGQQQIDSVTLEASGREDHDEQFGWHGTWQTAAGWEFIDGYRATLSYGTGFLAPSLGQQYG ATRFASFYGPGIASNPNLKPEESKQWEAGIDGLTGPLDWRISAYHYEIQNLIDYKNNQYINVKSATIKGL EWTGNVTTGPVEHHLTLQYVDPRDDDTGKVLYRRAKQQVKYELTGQIYALDWNVMYQYLGKRYDDDYDNG RDVKMGGLSLWDVGLSYPVTSHLTVRGKIANLFDKDYETVYGYQSAGREYTLSGSYTF

\section{>PAeLGP1}

MTLPFTRAAWRPLCSAAVLGAALWAAGASAAERRFDLPAQPLAASLSRLAQQAQVQVLFDESLLRGLRAP ALSGSYGVREALERLLVGSELELVEAGGGYVVRQRQVDAYSDNALQLDAQTIVGNGREVDASNVGRSTLT RRDIERQQADNIPSLLQTLPGVTMGGSPKPGGQTTNIWGLGDAEDVPYTLDGAQKSGFERYQQGTVFIEP EMIKRIEVEKGPHSVFTGNGGFGGTVHMETKDAPDLLREDRDLGAMLKYGYHSNDQQKIYSGAVFGRSED RRVDALLYLNGRDGRDMKLADNLPLSPTDYPINPKRLPNSAQDEKTGLFKLNLHPTEEHDLGFTYLRSKS SRWTPFSASSYPTPPSQWTIDRYGYELGLTRLLAHRDTTDTTWTGKYNYHPLDNPWIDLQLSYSDARTEQ LDRREDTAFYQLATGGKRMRTEYQDKVLELRNTSRFDTGALQHELTLGAALHKHKRDILMHMPGKTYETP RYNYGWLQPAFMPAGKQDTQSFYIQDAITYGSLTVTPSMRFDSVRNDGQANLAPIYDNPKLGHDYRAQTY SGWSPRLSVFWTATPNLAFFADYTETWRAPVIDEQYEVQNSSTIGGSSRDLDAERIHAIRGGSVINLPDL LVAGDSLQIRTTLFQNRIKDEIFRTRSVGCRQQSIDNGSIGGSCGDMLPLSNYRNLPGLTIKGFEIESFY DSQRLFGSLSYSWMTGKHDGAYSNPWGPNVWARDIPPPKWVAMLGLKVPEWDAKLGWQGEFVRKTDRLPS DRYSGGMGTGSGDIYWDHAANDSYDTHRLFAEWVPAKLGLKDTRIDFTVDNLFNRSYRQPLGGDLVYSQG RNAKISVTQFF

\section{$>$ YpeHasR}

MDNIIRDKKTIISVNKITTCILFALVTQGYSGQLAANTAETPTNNNDMALDKLNVEGKGNAHDSDWIYDE PRSVSEITREQLDNRPARHAADILEQTPGVYSSVSQQDPGLSINIRGIQDYGRVNMNIDGMRQNFMKSGH GQRNGSMYIDPEILSNVVIERGIFNGIGGAGAIGGIATFNTINASDFLAPEKELGGHIRAMTGDNGTRFI GSGALALGNPNGDILLAVSERNLKDYWPGNKGVLAGLRLYSPTRNVGDDLKNTKTLFTGYKMRSQLAKVG WNFEAGQRLEFSYLQTQIASPNASMLSEVLALSPSGKEITKIGWRNTSFTNVENRNIALDYRLNPEHISW LDATAKIYYVDTNDETDNANSLFKEYFWTQTRLKTRGLQLQNTNTFTPSDAHQIRLKYGLEWFSDKSEGY STRKLIERTTPPGKRAITSTFAQLNYEYDDWLRLEGGLRYDQFRLKGNTWLHTRSFLQPYTFENPCDRRI HEQSEKPGSRCSSRRPATMRWDVDRCEQQLSPTLAMGVKPGLEWLEFFGSYGKSWRPPAMTEVLATGTAH GYSWVLPNPFVAAERARTWEAGFNIQQSNLFIEDDHFAAKVAYFDTRIANYINLELGKAKPKFGGDSFTD VAYVNNLLKTRFRGLEYQLSYDAGTFYTNINYTRMIGVNNVCSPYAWLGGLQSVKYKYVGKVEQIYAVEN EVANNYVTCMNANVLFGSSAYLPGDRGSLTLGSRIFDRRLDFGTVIRYNKGYQDRSAQDENGNPLTAYVA DWPKYIVYDLYASYKVTNNLILRSSIENITNRAYLVNYGDTLSFAPSRGRTIQGGFEYKF

\section{>YpeIutA}

MKHKHLWVLNPCLLVMLTPAAWAEDQLVVSANRSHRSVAEMAQTTWVIEGQELEQQVQGGLEIKDILAQL IPGIDVSSQGRTNYGMNMRGRSIMVMIDGVRLNSSRSDSRQLDSIDPFNIAHIEVISGATSLYGGGRTGG LINIVTKKGQEGKQVELQIGGKTGFNSHNDHDENISAAMSGGTERAFGRFSVSYQRYGGWYDGKGNEVLI DNTQTGLQYSNRLDVMGTGTLNIDENQQLQLTTQYFNSESDGKHGLYLGQNFSAVTGTGQASNSAALNSD RIPGTERHLINLQYSNTDFWGQDLVAQVYYRDESLTFYPFPTLKDGKVSTIGASQQKTDFYGSKLTLNSE PIDSLTLTYGIDLEHESFNANQQFFNLAKAQQSGGMTLENAYNVGRYPSYTTTNLAPFLQTRYDINPIFT LSGGVRYQYTENKVDDFVGYAQQQAIASGSATSADPVPGGKTDYNNFLFNAGLLAHLTESQQTWFNFSQG FEIPDLAKYYGSGSYTLVNGHYQLQNSVNVNDSKLEGIKVDSYELGWRYTGDNLRTQMAGYYSLSDQTIS INKTDMTINVLPDKRRIYGVEGAVDYFFDNSEWSAGATFNLIKSETKVSGKWQKLTIDAASPSKATAYIG WAPGDWNLRVQSQQTFDVSDSKGDKIDGYNTIDFLSSYALPVGKLSFSIENLLDKEYTTVWGQRAPILYS PTYGSPNLYSYKGRGRTFGVNYSVLF

\section{>YpeFhuE}

MRILEKNHGKSRVRFNCTHPIKPAAWVLAANVSLLGCAYAATDENNSQKKERENNPANTTITVTASPLRH AGVTEDSGSYNTSSMSTATGLNISARETPQSVSVLTKQRMRDQNLNSVESAVNNITGISVRQFDSDRFGF TSRGMAVNNVMRDGVATFYDTRFNYGDNTLDTDMFDRIEVVRGAAGLMAGPGNPSAVINLVRKRPTQDFR GSVSAGVGSWEKWRTALDISGPLNSEGSVRGRFVTAYEDKNSFVNRYDQSKNPFYGILEIDVTPNTLFTF GADTQRTLTRGGMFGGLPLFNSAGGRTNYAQSATTASDWASAETRTQTLFSSLQHNFDNGWNIKGTFTFD NDKLRQDVMWPTGYPDPQTNIGMRPGSLSLIDGARRQQNYDIQVNGQYSLFGRQHQLGLGWNRQRQNIDN DYYLATCNATRTCPDLGDFTQPGWQYPKPVWSDKRAYGSKGRSDQSSQYVVTQLSLIDPLTLILGGRLTT WETRGDNFGTPQNARYKNEFVPYSGLTYDINRDLSVYTSYTEIFNPENRRDRNNTLLAPVSGQNYEAGLK 
GVAFDNSLDYSLAVFEIRQNNMPVPDTTAPRLPDNSQPYFAVDGTKTRGFEAEVSGKMTEDWNISAGYTQ YNVKLPSSNTQTPVTPVTPRKVLKLFTTYTLPGQLSDLTLGGGVNWQSQIARNLSSPIGIQQIGQGSFAI YSLMSRYQFTPQLSLTVNLDNLFNQHYYTQIGQYNQYLLGAPRNVDATVRYTF

\title{
>YpeFhuA
}

MNKINMFMLSTFMRSMFMRSTLIPSTFILSTLLLPAVVLAEDTIIVERNRSQTDSYQATTSVTANRSPAN IIDTPQNVTVVTHPVLEDYDVTNLGEALYFVSGITQSNTLGGTQDALIKRGFGNNRDGSILHDGIRSIQA RNFTPTSERVEVLKGPSSMLYGMNEPGGLINVISKKPQLDPHVHLEANASSFKGGGGQLDVTGPLGESGF AGRLIIDHQETDYWRNFGRNRQTVVAPSLDWYGENTTVHLAWERMEYLVPFDRGTVIDPRTGKPVDTPRD RRFDESYNATRGDQDTVTFSINHDLNEFWSTQLNYAFSRNSYSDNQARATAFNAETGVLTRQSDATANAK SHSQAVQWVVNGDLDWGFTHHQLMFGIDYEARRVFRGDMIRGSKNNGFNVYDPIYGTLPPSTAVSAADSD QRENIDSRALFIQDSMRLNEHWLLLGGLRYDSFDVMAGKGRPFKKNTDSSDSRLVPRAGVVYNLNDWSSL YVSYTESFKPNVSIATAIDALPPEYGKSWEAGYKIDINNRVTGTLAVYDIHKRNIMVSELVDGETVTRTA GKARSRGLELDMAGKVTDSLSLIGSYAYTDARLTEDPDNNGNDLPNVARHTAALFLSRDFGSTSLISGDE VKAGIGARYVGKRAGDAANSFWLDNYTVADAFIAWKMPLSGYQLKWQLNVKNLFDKTYYPSSANNLRIAI GEPRQVVLQGSIDF

\begin{abstract}
>YpeChuA
MLRSTSDRFRWSSLSLAIACTLPLATQAADTTTTQTSSKKHSTDTMVVTATGNERSSFEAPMMVTVIEGN APTSQTAATAADMLRQVPGLTVTGSGRTNGQDVVMRGYGKQGVLTLVDGVRQGTDTGHLNSTFLDPALVK RIEIVRGPAALLYGSGALGGVIAYETVDAADMLQPGQNSGYRVYSSAATGDHSFGLGASAFGRTDDLDGI LSFGTRDIGNIRQSNGFNAPNDETISNVLAKGTWQIDSIQSLSANLRYYNNSAIEPKNPQTSAPSSTNVM TNRSTIQRDAQLRYNIKPLDQEWLNATAQVYYSEVEINARPQGSAEEGREQTTEGVKLENRTRLFIESPA SHLLTYGTETYKQEQTPGGATESFPQAKIRFSSGWLQDEITLRDLPVSILAGTRYDNYSGSSDGYADVDA DKWSSRGAISITPTDWLMLFGSYAQAFRAPTMGEMYNDSKHFAIPIRPGLTLTNYWVPNPNLKPETNETQ EYGFGLRFSDLLMAEDDLQFKVSYFDTKAKDYISTRVDMQAMTTTSVNIDQAKIWGWDASMSYKTALFNW DLAYNRTRGKNQNTDEWLDTINPDTVTSIVDVPVANSGFSVGWIGTFANRSSRVSSSTPQAGYGVNDFYV SYKGQEAFKGMTTTMLLGNVFEKEYYTPQGIPQDGRNVKFFVSYQW
\end{abstract}

\section{$>$ YpeCir}

MAKVNQRFRKTHSAALVIAAVISSQGYAAEKTNPATPTDTMVVTASGFQQRIQDSAASISVVTREQIENK AYRDVTDALKDVPGVVITGGGSTSDISIRGMAAKYTLILVNGKRVDTRSTRPNSDGSGIEQGWLPPLAAI DRIEVVRGPMSSLYGSDAMGGVINIITRKVGKEWHGTVRADATLQEDSKSGDIFQTNAYASGPLIDGLLG LKVSGLLSHRSEDKI IDGYNQQRMRNGTATFTLTPDDNNEFDFDIGHYVQDRNSTPGRTLALNGTNSDTQ YDRNNYAVTHNGYYDFGNSTSYIQRDETRNPSRQMKSVDNIFNTQTSFLLDNHTLILGGQYRYEELYDKG NQLPSASDLKKLTRWSWALFAEDEWQMTNDFALTGGIRMDQDQNYGTHWTPRLYGVWHLADQWTLKGGVS GGYRSPDLRQATDDWGQLSGGGKGGLPALILGNSNLKPERSISQEIGILWDDQEGMNASVTLFYTDFKDK ITEVRNCDITTNTTGQCVFNGINYKFISDRINVDKAMTRGAEATFAWDINQAWSLATNYTFTQSEQKSGA FAGQPLNQMPKHMLNGTLNWKTTEDFATWIRANYRGKASEYLNRTSMGSRTPSYTFVDLGANYQLTKEVR LMGGVYNLLDKRVDIDVNDKVLDGRRYMVGASYDF

\section{>YpeBtuB}

MTIKKYTLLTALSVTAFSGWAQGNNTTDNNDEMVVTANRFPQPKSSVLAPVDVVTRADIDRWQSTNINDV LRRLPGVDIAQDGGMGQRSSLFIRGTNSSHVLVLIDGVRLNQAGITGASDLSQIPISLVQRIEYIRGPRS AVYGSDAIGGVINILTGRDKPGTTLSAGLGSNGYQTYDGSTQQKLGEDTTVTLAGNYTYSKGYDVVAGMP GAGGPRQPDRDGFMGKMLWAGLEHQFNEQFNGFARVYGFDNRSDYDGYTNYSNPLALIDTRKLSSRTYDT GLRYKNGIYASQFIASYNRTKDYNYSPLFGQHDITASLDEAEQYNLQWGNTFQLTNGMISAGADWQEQRT ERKSSNQNTTADFTQHNTGIYLTGQQQISDVTLEGAVRSDDNSQFGWHSTWQTSAGWEFIDGYRLIGSYG TAYKAPNLMQLYSAYGGNANLKPEKSKQWEGGVEGLTGPLTWRLSAYRNDIDQLIDYSNLTNGYFNINKA TIKGVEWTGSFDTGPLSHQVTLEYLDPRNADTHEILVRRAKQQVKYQLDWQVADLDWSVTYQYLGQRYDK DYSTYPEETVELGGVSLWDLAVSYPVTSHLTVRGRIANLFDKDYEMVYGYQTPGREYYFTGSYNF

\footnotetext{
>YpePsn

MKMTRLYPLALGGLLLPAIANAQTSQQDESTLVVTASKQSSRSASANNVSSTVVSAPELSDAGVTASDKL PRVLPGLNIENSGNMLFSTISLRGVSSAQDFYNPAVTLYVDGVPQLSTNTIQALTDVQSVELLRGPQGTL YGKSAQGGIINIVTQQPDSTPRGYIEGGVSSRDSYRSKFNLSGPIQDGLLYGSVTLLRQVDDGDMINPAT GSDDLGGTRASIGNVKLRLAPDDQPWEMGFAASRECTRATQDAYVGWNDIKGRKLSISDGSPDPYMRRCT DSQTLSGKYTTDDWVFNLISAWQQQHYSRTFPSGSLIVNMPQRWNQDVQELRAATLGDARTVDMVFGLYR QNTREKLNSAYDMPTMPYLSSTGYTTAETLAAYSDLTWHLTDRFDIGGGVRFSHDKSSTQYHGSMLGNPF GDQGKSNDDQVLGQLSAGYMLTDDWRVYTRVAQGYKPSGYNIVPTAGLDAKPFVAEKSINYELGTRYETA DVTLQAATFYTHTKDMQLYSGPVRMQTLSNAGKADATGVELEAKWRFAPGWSWDINGNVIRSEFTNDSEL YHGNRVPFVPRYGAGSSVNGVIDTRYGALMPRLAVNLVGPHYFDGDNQLRQGTYATLDSSLGWQATERMN
} 
>YPeLGP1

MISGKNKVCKKKLLACAVTSAISAFASNAIASNAIADTTSLAAMNRSAINISSTHNSNKKLNDADVITVT APLYSPLVVVTSPKVPRQPVPASDGTDYLRTIPGFSLVRNGGTNGDVVFRGMFGSRLKILTDGAEILGTC PSRMDAPTSYISPESFDLLTVVKGPQSVLWGPGVSAGTILFERERPHFDQSGVKGNASVLAGSNGRWDKN LDASLGNEQGYLRMMGNQSRAADYKDGNNVRVPSQWDKWNADMALGWTPDTDTLLELSVGKGNGEARYGG RGMDGSQFLRESLAARVEKSNIGEVLDKIEAKINYTYVDHIMDNKTLRTPPMMAMSSNLDRRMLGGRVMG TWLWQDLKLDAGTDMQTNTHRKNKQGNWDKNARFMNNGVFGELTWAASEQNKLISGARLDRHQVTNYTRA GEPTRSATLPAAFMRVEHNLANAPVMLYAGLGHTERFPDYWELFSPKFGPAGSTSAFEGVKSEKTTQIDI GGQYSGEQFRGWLSAYIGRVNDFILFKYDPKNLRISQADNINATIMGGEMGMEYALTPYWKADASLAYAW GENTRDNRPLPQIPPLEARLGLTFEAGDWSSSALWRLVNSQHRVAINEGNVVGKDFSSSAGFGVLSANVA YKVNKQVKLSSGIDNLLNKAYSEHLNLAGNSGFGYSVNTPLNEPGRTLWAKLNLTF

\section{>YPeLGP2}

MMVFYRKFFTVTVLISLPCLAWSQSHNNSKDELDTITVVAQKINQQQQKTPISISVLTGFDLERENIENI YESIMRIPNVYMVKAGNPSDAGFFTMRGTTPGMEGIQSVGFFIDGVYANTFDTELLDVDRIEVLRGPQAT LYGRNTESGVINVITKDPEFSPEYKIGLSYGNYNRTQVTTVLGGSINDSEQFSYRAALKYLYGNGYFKRD YDGKNNVDNLNDFSGRFKLRWQPMDDGWDVMTTFDIQNRRNGNTSFTALDKIKSGQKYVDSNYIGKSDVD AYKGQVNAVYTFDDIDFTSVSAYVDERKVDNQDLDFTRLSISELLMNRKTKQFSQEFRLNSKYSGPFNWL IGSYYFYQDDENEIDFRYLPYNLAQLRKSDIKTNNYAVFGNVNYYLLNDVELVAGARYDYEKKKLNFLMD NGFNPYQPYSHDNNSNSFGAFLPKVGLNYYITGDAMLYTSIARGYKSGGFNTLGPQSSRAYNAEYMTTYE AGVKTEWFDRTVRWNTSLFWNDMKDQQVEVAYYPISYSVNSGKSLSRGLESELAWRITRGLTVSANVGYT DAYFKNFPTEIKVDNNYIPVNYKGNRPANSPGYTYSIGADYNFLNGYFVNATYNVKGSTYLDNANSKKQP AYGLLDLTAGYENKDYGVNVWIKNILDETYVTRAFKMDDGIWYGRAGEPINFGVNFNVKF

\section{$>$ YPELGP3}

MSNKTIAFALVVASSAPVIAADNDNIMVVTASGYEQKIREAAASISVISQNELRQRNYNDLAQALSDVEG VDVNSSTGKTGGLDISIRGMPSAYTLILVDGIRQNGTSDVTPNGFGAMNTSFMPPLSAIERIEVIRGPMS TLYGSDAIGGVVNIITKKITKAWASSATLEHTFQENTAFGDSSKFSFYSSGPAVEDQLGLSLRGTIFRRD ASRVESSNTGVELSRRGPNPVKADNYNLGGKLFWQLNTQSTLWLDGDIANQKYDNSANQLGTIGARGGYE DTLRYQRRKITLGNDNRLDFGTWNSSLSYNQTENIGRLITNASVPQGSGLAGEKRLLKNTNIILDSKLVA PLGDNHMVTLGGQYWNAIMKDGIVLANNGDEFAQDAWSLFSEDEWRLLDSLALTYGARYEYQTTFGGHIS PRAYLVWDAQDNWTVKGGVSTGYKTPTLAQLHNGISGVTGQGTITTIGNPKLEPESSVNTEVGVYYENET GFGANVTLFHNRFRNKINSVSIDNTTSTYTNVGKAITQGIEVASTIPLWSDDWMLGINYTFTDSEQKDGN NKGARLTNTPKNMVNARLNWNINEQLSTWLKAEYRSKTARFTQNYANLSAANKVVYNNLGSEFKPFSVLN LGVAYKVTKDVTLNGAVNNLLDKDFTRTHIFAVGNGTTTAGDYFTSSQSTAGYVVPGRNYWVSVNVNF

\section{$>$ SmaHasR}

MFIHKGTTPAGRLATAVRAALAAMMLTQPAVALAAQAEASSAQAAQQKNFNIAAQPLQSAMLRFAEQAGM QVFFDEVKLDGMQAAALNGSMSVEQGLRRLIGGNPVAFRLQPQGQIVLSRLPTANGDGGALALDSLTVLG AGGNNANDWVYDEPRSVSVISREQMDNRPARHAADILEQTTGAYSSVSQQDPALSVNIRGIQDYGRVNMN IDGMRQNFQKSGHGQRNGTMYIDSELLSGVTIDKGTTGGMGSAGTLGGIATFNTVSASDFLAPGKELGGK LHASTGDNGTHFIGSGILALGNETGDILLAASERHLGDYWPGNKGDIGNIRINNDTGNYDRYAESIKNNK IPDTHYRMHSRLAKVGWNLPANQRLQLSYLQTQTASPIAGTLTNLGTRPPYELGWKRTGYTDVMARNAAF DYSLAPEDVDWLDFQAKLYYVDTQDDSDTYSTSSLLDNGYATRTRLRTYGAQAQNTSRFSLAPGHDFRAN YGLEFYYDKATSDSSRQGMEGVTPAGNRSVASLFANLTYDYDGWLTLEGGLRYDRYRLRGQTGLSYPDLA KDGQRYTIDNPCKALRLTGCSTTTREDWDVDRDQGKLSPTLAVAVRPGVEWLELYTTYGKSWRPPAITET LTNGSAHSSSTQYPNPFLQPERSRAWEVGFNVQQPDLWFEGDRLVAKVGYFDTKVDNYINLAIDRNKPGL VQPSIGNAAYVNNLSKTRFRGLEYQLNYDAGVFYADLTYTHMIGKNEFCSNKAWLGGRLRYGDGSRRGNF YVEPDAASNDFVTCDGGTQFGSAAYLPGDRGSVTLGGRAFDRKLDAGVTVRFAPGYQDSSVPSNYPYLAD WPKYTLFDLYASYKLTDSLTLRGSVENLTNRAYVVSYGETLANTLGRGRTVQGGVEYRF

\footnotetext{
$>$ BpeFauA

MPDPAHFSPAHSRRLRFFAHVLHLLGALFTQHALAQEARTGNDIAQLPAISVTGREISDLTEGTNAYTTE AMSTATGLTLSPRETPQSVSVVTRQQIEDQGLTDTGAILATAPGISVTRSDSNRYSFSARGFTIDNFQFD GLVSPILSQWNYGSTDMDAAIYDHVEIVRGATGLMTGSGNPSAAVNFVRKRPLREFAATFNASVGSWDYV RGDADISVPITEDGRIRSRLVAAYSQGDSYVHFLDTRRRTFYGVVSADLTPDTVLTTSVEYQHNHSNGFG SGFPLFYSDGSRTDFNRSVANNAPWARQDTEATTYFVDLTHRFTNDWKLRAAYSHTDGRYLMKHVYRGGY PDRHTGIIAAPPAFSNYDGNLDRDDIHFSLSAPFEAFGLRHEVALGWMSIDNHSDIQRYAMVGPAPAIGS FFDWRRAHIQEPSWADTLSPADDVRTKQTGAYLVGRFALAEPLHLIVGDRWSDWKTKQMYFGSRREYRIK NQFTPYAGLTYDINDTYTAYASYTEIFQPQNARDTSGGILPPIKGKSYELGLKAAYLEGRLNTSAALFQT
} 
RQDNLAQVIPGSSIPGFPNMQASRAASGAKVEGIDLEASGQILPDWNIGASYTHFTTKDASGNPINTNHP RSLFKLYTTYRLPGALHRLTVGGGVDWQSRMYQAAASPRGNVEVEQDSYALVSLMARFDFNKKLSATLNV NNLFDKKYYDQIGFYSQGWWGAPRNVMLNLRAQY

$>$ NmeFrpB

MNTPLFRLSLLSLTLAAGFAHAAENNAKVVLDTVTVKGDRQGSKIRTNIVTLQQKDESTATDMRELLKEE PSIDFGGGNGTSQFLTLRGMGQNSVDIKVDNAYSDSQILYHQGRFIVDPALVKVVSVQKGAGSASAGIGA TNGAIITKTVDAQDLLKGLDKNWGVRLNSGFASNEGVSYGASVFGKEGNFDGLFSYNRNNEKDYEAGKGF RNNFNGGKTVPYSALDKRSYLAKIGTSFGDGDHRIVLSHMKDQHRGIRTVREEFTVGGDKERISMERQAP AYRETTQSNTNLAYTGKNLGFVEKLDANAYVLEKERYSADDSGTGYAGNVKGPNHTQITTRGMNFNFDSR LAEQTLLKYGINYRHQEIKPQAFLNSQFKIEDKEKATDEEKNKNRENEKIAKAYRLTNPTKTDTGAYIEA IHEIDGFTLTGGLRYDRFKVKTHDGKTVSSNNLNPSFGVIWQPHEHWSFSASHNYASRSPRLYDALQTHG KRGIISIADGTKAERARNTEIGFNYNDGTFAANGSYFWQTIKDALANPQNRHDSVAVREAVNAGYIKNHG YELGASYRTGGLTAKVGVSHSKPRFYDTHKDKLLSANPEFGAQVGRTWTASLAYRFQNPNLEIGWRGRYV QKAVGSILVAGQKDRNGKLENVVRKGFGVNDVFANWKPLGKDTLNVNLSVNNVFNTFYYPHSQRWTNTLP GVGRDVRLGVNYKF 